\title{
Evolution and instability of unsteady nonlinear streaks generated by free-stream vortical disturbances
}

\author{
By PIERRE RICCO ${ }^{1}$, JISHENG LU O ${ }^{2}$ \\ AND XUESONG W U ${ }^{3} \dagger$ \\ ${ }^{1}$ Division of Engineering, King's College London, London WC2R 2LS, UK \\ ${ }^{2}$ Department of Mechanics, Tianjin University, Tianjin 30072, China \\ ${ }^{3}$ Department of Mathematics, Imperial College London, London SW7 2BZ, UK
}

(Received 13 January 2011)

We investigate the influence of free-stream vortical disturbances on the evolution and instability of an incompressible laminar boundary layer, focusing on components of sufficiently long wavelength, which are known to penetrate into the boundary layer to generate streamwise elongated streaks. The free-stream disturbance is assumed to be sufficiently strong (but still of small amplitude) that the induced streaks acquire an $\mathcal{O}(1)$ streamwise velocity in the region where the boundary-layer thickness becomes comparable with the spanwise wavelength of the perturbation. The formation and evolution of the streaks are governed by the nonlinear unsteady boundary-region equations supplemented by appropriate upstream and far-field boundary conditions. This initial-boundary-value problem is solved for the special case where the free-stream disturbance is modelled by a pair of oblique vortical modes with the same frequency but opposite spanwise wavenumbers. Nonlinearity is found to inhibit the response. The nonlinear interaction alters the meanflow profile appreciably, the shape of which is in quantitative agreement with experimental measurements. Wall-normal inflection points are detected in the instantaneous streamwise velocity profiles. The secondary stability analysis indicates that in the presence of free-stream disturbance with an intensity of $2.8 \%$, the resulting streaky boundary layer becomes inviscidly unstable. The characteristic frequency, phase and group velocities, and growth rate of unstable sinuous modes are found to be in broad agreement with recent experiments. The present theoretical framework allows in principle a quantitative relation to be established between the characteristics of free-stream turbulence and the secondary instability, and this relation may be exploited to develop an efficient and physics-based approach for predicting bypass transition.

\section{Introduction}

Laminar-turbulent transition in a boundary layer is crucially influenced by free-stream turbulence (FST). As the intensity of FST, Tu, is increased, not only does the transition location shift upstream significantly (Dryden 1958), the inherent mechanisms may be fundamentally different. When $T u$ is relatively low $(T u<0.1 \%)$, transition is caused by the viscous Tollmien-Schlichting (T-S) instability (Schubauer \& Skramstad 1947; Kachanov 1994). In this case, FST affects transition primarily through receptivity (Goldstein \& Hultgren 1989; Saric et al. 2002), in which FST excites unstable modes either due to

$\dagger$ Email address for correspondence: x.wu@ic.ac.uk 
the adjustment by the nonparallelism of the base flow near the leading edge (Goldstein 1983; Ricco \& Wu 2007), or by interacting with local or distributed steady inhomogeneity (Goldstein 1985; Ruban 1985; Duck et al. 1996; Wu 2001a,b). The observed upstream shift of the transition location with $T u$ may be attributed to the increased initial amplitudes of unstable modes. FST of moderate intensity may influence the growth rates of T-S waves directly, but apparently contradictory findings have been reported. For example, experimental studies of Kendall $(1991,1998)$ indicate that FST enhances the amplification of T-S waves, and this destabilizing effect was demonstrated and explained by the theory of Wu \& Choudhari (2003). However, Boiko et al. (1994) observed that T-S waves amplify at a reduced rate in a boundary layer perturbed by FST. A similar stabilizing effect of roughness-induced steady streaks has been reported by Fransson et al. (2006), and the theoretical support was provided by Cossu \& Brandt (2002) and Cossu \& Brandt (2004).

In the presence of relatively high level of FST $(T u>1 \%)$, transition apparently occurs without involving T-S waves. Instead low-frequency disturbances appear to penetrate into the boundary layer and amplify significantly to distort the flow in a three-dimensional manner (Dryden 1936; Taylor 1939). The resulting boundary layer features streamwise elongated, spanwise alternating low- and high-speed regions (Klebanoff 1971), which are referred to as streaks or Klebanoff modes (Arnal \& Juillen 1978; Kendall 1991; Westin et al. 1994). Experiments indicate that streaks may become unstable and break down to form sporadic turbulent spots (Matsubara \& Alfredsson 2001), which grow and merge, leading to fully developed turbulence. Streak instability appears to be inviscid, developing over a length scale much shorter than that pertaining to the viscous T-S instability. Quantitative data about the characteristics of streak instability are however scarce because detailed measurements where free-stream disturbances were introduced in a controlled manner have not yet been conducted. Nevertheless, recent laboratory observations, carried out in uncontrolled conditions, find that the propagation speed of unstable modes is about $0.8 U_{\infty}$, and growth rates are about $0.01 U_{\infty} / \delta^{*}$ (Mans et al. 2007), where $U_{\infty}$ is the free-stream velocity and $\delta^{*}$ the local boundary-layer displacement thickness.

On the basis of the discussions above, it may be concluded that a thorough understanding and the eventual prediction of bypass transition require investigations of three related processes: (a) the entrainment of FST into the boundary layer, (b) amplification and formation of streaks, and (c) the secondary instability of streaks.

Much effort in the last a few decades has been directed to (b). The central mechanism is the so-called transient growth. The idea originated from the observation of Ellingsen \& Palm (1975) that in the inviscid limit three-dimensional disturbances of infinite streamwise wavelength on a shear flow may amplify algebraically in that the streamwise velocity grows proportionally to time; see also Landahl (1980). When the viscous effect is included, three-dimensional disturbances may undergo considerable transient growth before being attenuated by viscosity, provided that their streamwise wavelength is sufficiently long (Hultgren \& Gustavson 1981; Butler \& Farrell 1992). Standing waves consisting of pairs of such disturbances with opposite spanwise wavenumbers manifest as streaks. Mathematically, the transient growth may be attributed to the non-normality of the linear operator governing the evolution of the perturbation (Trefethen et al. 1993). More recently, it was shown that three-dimensional disturbances of a similar form may exhibit substantial spatial transient growth with the downstream distance in boundary layers (Luchini 1996, 2000; Tumin 2001). Initial or inflow perturbations that give rise to maximum linear transient growth have been identified, and the induced boundary-layer signature is referred to as the optimal disturbance (Andersson et al. 1999). Zuccher et al. (2006) used a nonlinear optimization procedure to select the initial disturbance which leads to maximum 
nonlinear growth. Optimal disturbances offer an upper bound of possible growth, but it should be recognized that they are not streaks generated by physically realizable external disturbances. Non-optimal steady or nearly steady three-dimensional disturbances are likely to be amplified by a factor of similar order of magnitude since transient growth is due to the generic and robust mathematical property (i.e. non-normality) of the operator governing the perturbation rather than to a delicate dependence on the initial condition.

Secondary instability of steady streaks has been considered by several investigators. Andersson et al. (2001) analysed the stability of streaks modelled by an optimal disturbance. They find that streaks may support both sinuous and varicose modes. The sinuous mode instability was found to be more dangerous, and occurs when the streak amplitude, measured by its streamwise velocity, exceeds a threshold $0.26 U_{\infty}$. The instability has been shown to be of convective nature (Brandt et al. 2003).

Bypass transition has also been studied by means of direct numerical simulations (DNS). Brandt \& Henningson (2002) simulated breakdown of streaks developed from an optimal disturbance. Jacobs \& Durbin (2001) performed DNS of bypass transition triggered by FST, which was represented by superposition of continuous spectra of the Orr-Sommerfeld and Squire operators. They found that streaks are generated within the boundary layer but remain stable, and breakdown does not occur until streaks are lifted up to the edge of the boundary layer. Using a similar model for FST, Brandt et al. (2004) investigated the influence of the length scale and spectral property of FST on streak formation. Their work indicates that sinuous and varicose instabilities operate to cause transition. The respective roles of low- and high-frequency components of FST in bypass transition were studied by Zaki \& Durbin (2006). Their simulations show that transition does not take place when the free-stream disturbance is composed solely of a low or a high frequency component even though streaks are generated in the former case. Transition occurs only when both components are simultaneously present in the FST, suggesting that low-frequency components play the role of distorting and destabilizing the boundary layer, while high-frequency components are required to trigger unstable modes. Nagarajan et al. (2007) and Ovchinnikov et al. (2008) performed DNS of bypass transition in boundary layers over plates with an elliptic leading edge, focusing respectively on the role of the bluntness and the integral length scale of FST. It was found that bypass transition was indeed associated with streak breakdown when the FST intensity is low and the leading edge is relatively sharp, or when the integral length scale (normalized by the length of the major axis of the ellipse) is small. However, when either of the FST intensity, bluntness and integral length is increased sufficiently, turbulent spots form spontaneously apparently without exhibiting any connection with streaks. The underlying physical mechanisms of the alternative processes remain a mystery, but see Goldstein \& Sescu (2008), where a possible explanation for the observation was proposed.

In summary, it may be concluded that the transient growth theory, secondary instability analysis and related DNS have shed important light on various aspects of bypass transition. However, the current understanding and description of transition remain incomplete and inadequate for achieving the ultimate goal of studying bypass transition, which is to predict (i) the quantitative relation between the transition location and the intensity (and other relevant statistical properties) of FST, and (ii) the threshold of FST which delineates the usual T-S and bypass transition routes. The theory of transient growth provides a mathematical explanation for the development of streamwise vortices which are already present within the boundary layer, but the vital link of these perturbations with FST is missing since FST, which causes bypass transition in the first place, is not accounted for in the formulation. Use of optimal disturbances as a model 
for streaks in secondary instability calculations cannot possibly provide an answer to the central question of practical interest, which is how streak instability or bypass transition is quantitatively related to FST. Modelling FST as a superposition of continuous spectra of the Orr-Sommerfeld and Squire operators represents a significant progress, but this approach ignores the crucial entrainment process in the region near the leading edge, where non-parallelism plays a leading-order role for relevant long-wavelength components. The stability properties and breakdown characteristics revealed in most previous studies are therefore only of qualitative value. For a quantitatively accurate prediction, it is necessary to take an integrated approach, in which FST is properly specified and its entire entrainment process into the boundary layer is satisfactorily described. In DNS, this may be achieved by imposing FST well upstream of the leading edge as was done, e.g. in Ovchinnikov et al. (2008). However, the resulting extended computation domain renders DNS an excessively expensive tool for systematic parametric studies.

Disturbances present in the oncoming free stream may be of different form, including

(a) Spanwise variation of an otherwise uniform mean stream (with the corresponding vorticity of the disturbance being normal to the plat);

(b) Steady streamwise vorticity superimposed on the mean flow;

(c) Unsteady vortical fluctuations of the convected-gust type.

The parameters characterizing these disturbances are the amplitude $\epsilon$, the Reynolds number $R_{\Lambda}=U_{\infty} \Lambda / \nu$ and the typical frequency $k_{1}$ (normalized by $U_{\infty} / \Lambda$ ), where $\Lambda$ is the spanwise integral length scale of FST.

The experiments of Klebanoff \& Tidstrom (1959) and Bradshaw (1965) indicated that the Blasius boundary layer is remarkably sensitive to disturbances of type (a). Prompted by this finding, Crow (1966) employed the linearised boundary-layer equations to calculate the boundary-layer response to such small-amplitude disturbances. He found that the streamwise velocity within the boundary layer grows linearly with the downstream distance $x$. It may be noted that the homogeneous system admits an asymptotic eigen solution, whose streamwise velocity grows algebraically, i.e. $u \sim x^{0.216}$ (Luchini 1996). Since this growth is much slower than that of Crow's forced solution, only the latter is of significance in the subsequent development. Goldstein et al. (1992) pointed out that the forced motion remains linear only within a distance much smaller than $\min \left\{R_{\Lambda}, \epsilon^{-1}\right\}$ to the leading edge. The continued development downstream is nonlinear, governed by the nonlinear boundary-layer equations, if $\epsilon R_{\Lambda} \gg 1$, or by the so-called nonlinear boundaryregion equations which include cross-flow ellipticity if $\epsilon R_{\Lambda}=\mathcal{O}(1)$, as was shown by Goldstein \& Wundrow (1998). Numerical solutions reveal that nonlinearity leads to a strong vorticity concentration along the spanwise direction causing the viscous boundary layer to separate for $\epsilon R_{\Lambda} \gg 1$, but the streamwise velocity profile of the distorted boundary layer does not become inflectional. In the same vein, Goldstein \& Leib (1993) and Wundrow \& Goldstein (2001) investigated nonlinear streaks driven by disturbances of type (b), streamwise vortices, and found that an inflection point appears on the boundarylayer profile before separation occurs. The distorted flow thus supports short-wavelength inviscid Rayleigh instability, which was linked to bypass transition to turbulence.

FST is generally considered to consist primarily of unsteady disturbances of type (c), i.e. vortical waves propagating at the speed of the free stream. Of particular relevance are low-frequency (long-wavelength) components with $k_{1} \ll 1$. Their entrainment into the boundary layer and the development of the induced streaks are governed by the boundaryregion equations if $k_{1}=\mathcal{O}\left(R_{\Lambda}^{-1}\right)$, as was shown by Leib et al. (1999a) (referred to as LWG hereafter). These equations may be linearised if the intensity of FST is sufficiently weak, i.e. $\epsilon \ll R_{\Lambda}^{-1}$. Numerical solutions indicate that the boundary layer acts as a filter, allowing low-frequency perturbations to penetrate into the boundary layer while the 


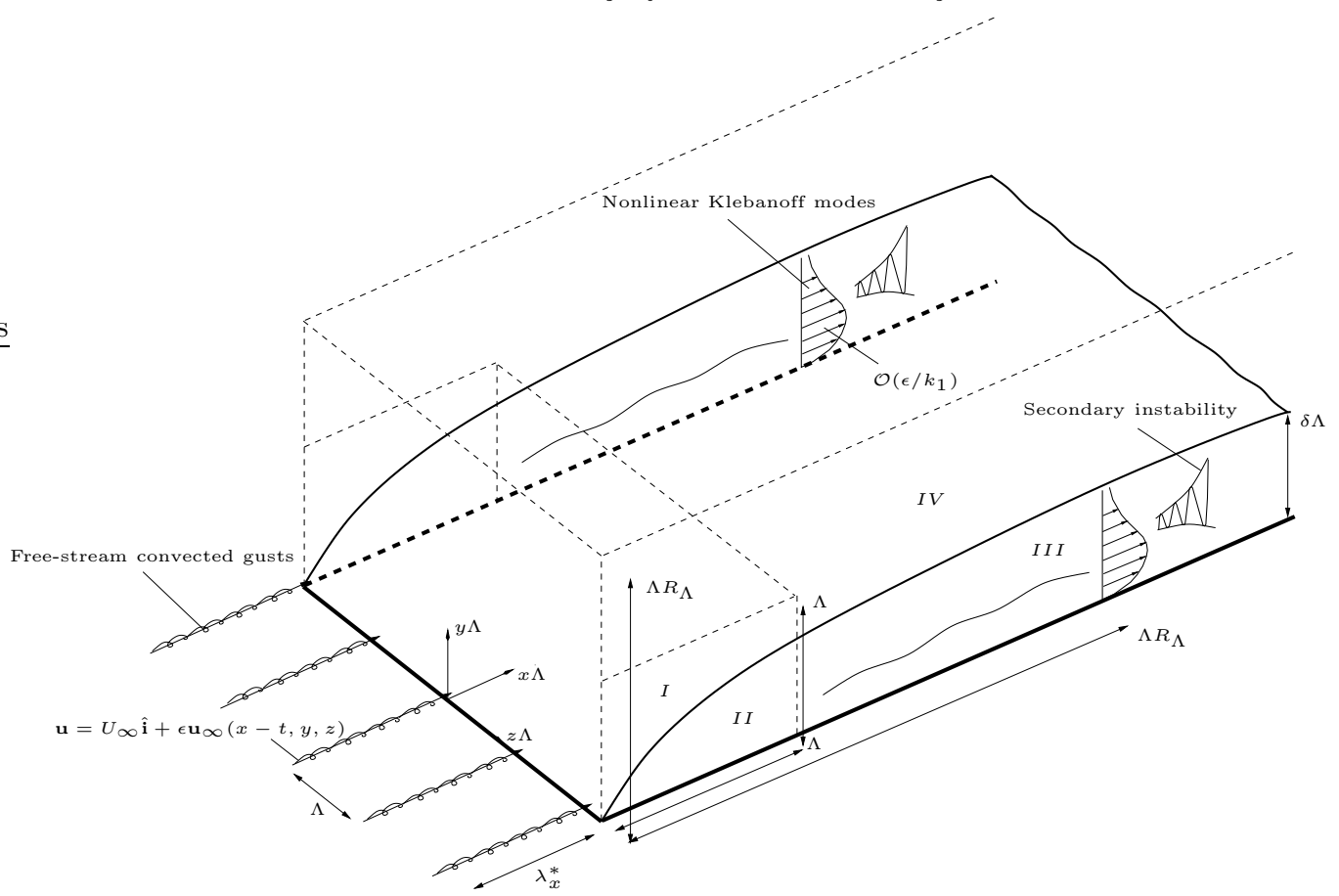

FiguRE 1. A schematic illustration of the problem under consideration, the coordinate system and the flow regimes.

high-frequency ones are absorbed in the outer edge of boundary layer. For higher level FST with typical intensity $\epsilon=\mathcal{O}\left(R_{\Lambda}^{-1}\right)$, the fully nonlinear boundary-region equations have to be reinstated because streaks attain an order-one amplitude.

Wu \& Choudhari (2003) are among the first to investigate the impact of FST-induced unsteady streaks on boundary-layer instability. They considered the limiting case $R_{\Lambda}^{-1} \ll$ $k_{1} \ll 1$ and $\epsilon \ll R_{\Lambda}^{-1}$, for which streaks can be approximated by the linear boundary-layer solution because they attain the maximum magnitude but remain linear in the region where the boundary-layer thickness $\delta^{*} \ll \Lambda$. Due to the inherent unsteadiness, streaks of moderate amplitude may alter the near-wall curvature of the boundary layer by an $\mathcal{O}(1)$ amount, thereby enhancing the amplification rate of T-S waves. When the distortion exceeds a certain threshold, the perturbed mean profile develops an inflection point during certain phases of the streak modulation, and thus supports intermittent inviscid sinuous and varicose instability modes. The importance of unsteadiness is further highlighted by the recent work of Goldstein \& Sescu (2008). They found that in contrast to the steady limit (Goldstein et al. 1992), unsteady disturbances of type (a) cause the nonlinearly distorted profile to become inflectional, and therefore render the flow inviscidly unstable.

In typical experiments and applications (such as turbomachinery), the FST level is likely to be sufficiently high that fully developed streaks are nonlinear, and appear in the region where $\delta^{*}=\mathcal{O}(\Lambda)$. The present work therefore investigates the development and instability of streaks in the generic regime corresponding to $\epsilon=\mathcal{O}\left(R_{\Lambda}^{-1}\right)$ and $k_{1}=$ $\mathcal{O}\left(R_{\Lambda}^{-1}\right)$ as $R_{\Lambda} \rightarrow \infty$, for which streaks acquire an $\mathcal{O}(1)$ streamwise velocity when $\delta^{*}=$ $\mathcal{O}(\Lambda)$.

The rest of the paper is organized as follows. In $\S 2$, we formulate the problem by specifying the free-stream disturbance and scaling relations. The flow structure proposed for steady free-stream disturbance (Wundrow \& Goldstein 2001) are directly applicable to 
low-frequency disturbances. The mathematical problem consists of the unsteady nonlinear boundary-region equations (UNBREs), and appropriate outer boundary conditions and upstream conditions near the leading edge, which account for the action of the freestream disturbance on the boundary layer. In $\S 3$, we describe the numerical procedure to solve the UNBREs. The results are presented in $\S 4$. The instability of nonlinear streaks is analysed in $\S 5$. A summary and concluding remarks are given in $\S 6$.

\section{Problem formulation: scaling and equations of motion}

We consider the incompressible two-dimensional flow due to a uniform velocity $U_{\infty}$ past an infinitely thin flat plate. Superimposed on the oncoming stream are small-amplitude fluctuations, which are represented by vortical modes of the convected-gust type (see figure 1). The analysis is pertinent to the special case of a pair of free-stream vortical modes with the same frequency (and hence streamwise wavenumber), but opposite (dimensional) spanwise wavenumbers $\pm k_{3}^{*}$; it is hoped that experiments in which disturbances of this simple form are generated in a controlled manner would become possible in the near future. The extension to general homogeneous, statistically-stationary FST is a subject of our ongoing investigations.

The flow is described in a Cartesian coordinate system, in which a point is represented by a vector $\mathbf{x}=x \hat{\mathbf{i}}+y \hat{\mathbf{j}}+z \hat{\mathbf{k}}$, where $\hat{\mathbf{i}}, \hat{\mathbf{j}}$ and $\hat{\mathbf{k}}$ are the unit vectors in the streamwise, wallnormal and spanwise directions, respectively. The inverse of the spanwise fundamental wavenumber $k_{3}^{*}$ of the free-stream gust is taken to be a reference length $\Lambda=1 / k_{3}^{*}$, and hence $k_{3}=1.0$. The time $t^{*}$ is normalized by $\Lambda / U_{\infty}$. The velocities and pressure $p^{*}$ are made dimensionless by $U_{\infty}$ and $\rho U_{\infty}^{2}$, respectively.

The disturbance in the upstream region is passively advected by the background mean flow, and it can be written as

$$
\mathbf{u}-\hat{\mathbf{i}}=\epsilon \mathbf{u}_{\infty}(x-t, y, z)=\epsilon\left(\hat{\mathbf{u}}_{+}^{\infty} \mathrm{e}^{\mathrm{i} k_{3} z}+\hat{\mathbf{u}}_{-}^{\infty} \mathrm{e}^{-\mathrm{i} k_{3} z}\right) \mathrm{e}^{\mathrm{i} k_{1}(x-t)+\mathrm{i} k_{2} y}+\text { c.c. },
$$

where $\epsilon \ll 1$ measures the amplitude of the oncoming perturbation, $\mathbf{k}=\left\{k_{1}, k_{2}, k_{3}\right\}$, and $\hat{\mathbf{u}}_{ \pm}^{\infty}=\left\{\hat{u}_{1, \pm}^{\infty}, \hat{u}_{2, \pm}^{\infty}, \hat{u}_{3, \pm}^{\infty}\right\}=\mathcal{O}(1)$. From the continuity equation, it follows that

$$
k_{1} \hat{u}_{1, \pm}^{\infty}+k_{2} \hat{u}_{2, \pm}^{\infty} \pm k_{3} \hat{u}_{3, \pm}^{\infty}=0 .
$$

The ensuing evolution of the disturbance outside and within the boundary layer depends on $k_{1}, \epsilon$ and the Reynolds number

$$
R_{\Lambda}=U_{\infty} \Lambda / \nu
$$

where $\nu$ is the kinematic viscosity of the fluid. As in LWG, we assume that $R_{\Lambda}$ is asymptotically large, i.e. $R_{\Lambda} \gg 1$, and consider long-wavelength disturbances with

$$
k_{1}=\mathcal{O}\left(R_{\Lambda}^{-1}\right) .
$$

The disturbance remains linear when the turbulent Reynolds number is $r_{t}=\epsilon R_{\Lambda} \ll 1$. We now take

$$
r_{t}=\mathcal{O}(1)
$$

for which the disturbance undergoes nonlinear development. For steady free-stream disturbances, it has been shown by Goldstein (1997) that the flow domain can be divided into four distinct asymptotic regions. The same structure holds for the present unsteady (but low-frequency) disturbances of interest, and is shown in figure 1 . In the inviscid region-I over $\mathcal{O}(\Lambda)$ distances from the leading edge (i.e. $x=\mathcal{O}(1), y=\mathcal{O}(1)$ ) and in the viscous boundary layer (region-II), the disturbance is linear as in LWG. Two distinct 
regions emerge further downstream $\left(x=\mathcal{O}\left(R_{\Lambda}\right)\right)$, an inner region-III which has a width of $\mathcal{O}(\Lambda)$ and an outer region-IV whose transversal scale is of $\mathcal{O}\left(\Lambda R_{\Lambda}\right)$. The main focus of the present analysis will be on these two regions, in which nonlinear effects come into the play and streaks may undergo secondary instability.

\subsection{Disturbances in the outer region}

The outer region corresponds to $x=\mathcal{O}\left(R_{\Lambda}\right)$ and $y=\mathcal{O}\left(R_{\Lambda}\right)$, and so we introduce

$$
\bar{x}=k_{1} x, \quad \bar{y}=k_{1} y
$$

in view of (2.3). The perturbation in this region consists not only of the three-dimensional vortical disturbance convected from upstream, which depends on the relatively short transverse variable $y$, and but also of the two-dimensional disturbance that is induced by the viscous motion within the boundary layer through the displacement effect. The latter arises because the streamwise velocity of the boundary-layer signature acquires an $\mathcal{O}(1)$ amplitude, and attenuates over a large transverse distance. The situation is analogous to that for the steady perturbations considered by Wundrow \& Goldstein (2001). Following them, we decompose the solution as

$$
\left.\begin{array}{rl}
u & =1+\epsilon\left[\bar{u}_{0}(\bar{x}, \bar{y}, \bar{t})+\hat{u}_{0}(\bar{x}, \bar{y}, y, z, \bar{t})\right]+\ldots, \\
v & =\epsilon\left[\bar{v}_{0}(\bar{x}, \bar{y}, \bar{t})+\hat{v}_{0}(\bar{x}, \bar{y}, y, z, \bar{t})\right]+\ldots, \\
w & =\epsilon \hat{w}_{0}(\bar{x}, \bar{y}, y, z, \bar{t})+\ldots, \\
p & =-\frac{1}{2}+\epsilon \bar{p}_{0}(\bar{x}, \bar{y}, \bar{t})+\epsilon^{2} \hat{p}_{1}(\bar{x}, \bar{y}, y, z, \bar{t})+\ldots,
\end{array}\right\}
$$

where $\bar{t}=k_{1} t=\mathcal{O}(1)$. The terms $\bar{u}_{0}, \bar{v}_{0}$ and $\bar{p}_{0}$ represent the displacement-induced inviscid flow, and depend only on the slow streamwise and transverse variables $\bar{x}$ and $\bar{y}$. This two-dimensional part is (taken to be) governed by the linearised unsteady Euler equations

$$
\bar{u}_{0 \bar{x}}+\bar{v}_{0 \bar{y}}=0, \quad \bar{u}_{0 \bar{t}}+\bar{u}_{0 \bar{x}}=-\bar{p}_{0 \bar{x}}, \quad \bar{v}_{0 \bar{t}}+\bar{v}_{0 \bar{x}}=-\bar{p}_{0 \bar{y}},
$$

subject to the boundary condition

$$
\bar{u}_{0}, \bar{v}_{0} \rightarrow 0 \text { as } \bar{y} \rightarrow \infty
$$

By matching $\epsilon \bar{v}_{0}$ with the spanwise averaged normal velocity in the boundary layer given by (2.27), the other boundary condition may be derived as

$$
\bar{v}_{0}=\left(k_{1} / \epsilon\right) \bar{\delta}_{\bar{x}}(\bar{x}, \bar{t}) \text { at } \bar{y}=0
$$

where $k_{1} / \epsilon=\mathcal{O}(1)$ for the scaling adopted, and $\bar{\delta}(\bar{x}, \bar{t})$ denotes the spanwise averaged displacement thickness, the precise definition of which will be given later when the flow in region-II is considered (see (2.28)). Elimination of the pressure from (2.6) shows that the vorticity $\bar{\omega}_{0} \equiv\left(\bar{u}_{0 \bar{y}}-\bar{v}_{0 x}\right)$ satisfies the equation $\left(\partial_{\bar{t}}+\partial_{\bar{x}}\right) \bar{\omega}_{0}=0$, so that $\bar{\omega}_{0}=\bar{\omega}_{0}(\bar{x}-\bar{t})$. It can be inferred that $\bar{\omega}_{0} \equiv 0$ since $\bar{\omega}_{0} \rightarrow 0$ as $\bar{x} \rightarrow 0$, implied by the condition that both $\bar{u}_{0}$ and $\bar{v}_{0}$ vanish upstream. The two-dimensional flow is therefore irrotational with $\bar{v}_{0}$ and $\bar{u}_{0}$ satisfying the Laplace equation. The relevant solution can be obtained by using complex variable theory as (cf. Wundrow \& Goldstein (2001))

$$
\bar{u}_{0}=-\Re\left[\mathrm{i}\left(k_{1} / \epsilon\right) \bar{\delta}_{\bar{\xi}}(\bar{\xi}, \bar{t})\right], \quad \bar{v}_{0}=\Re\left[\left(k_{1} / \epsilon\right) \bar{\delta}_{\bar{\xi}}(\bar{\xi}, \bar{t})\right],
$$


where $\bar{\delta}(\bar{\xi}, \bar{t})$ is the analytic continuation of $\bar{\delta}(\bar{x}, \bar{t})$ to the complex plane $\bar{\xi}=\bar{x}+\mathrm{i} \bar{y}$. From the momentum equations (or the Bernoulli's equation), the pressure is found to be

$$
\bar{p}_{0}=\Re\left[\mathrm{i}\left(k_{1} / \epsilon\right)\left(\bar{\delta}_{\bar{\xi}}(\bar{\xi}, \bar{t})+\bar{\delta}_{\bar{t}}(\bar{\xi}, \bar{t})\right)\right] .
$$

The result (2.9)-(2.10) generalizes (3.16) of Wundrow \& Goldstein (2001) to the unsteady case.

It is worth noting that the decomposition (2.5) and the specification of $\left(\bar{u}_{0}, \bar{v}_{0}, \bar{p}_{0}\right)$ via (2.6)-(2.8) are based primarily on physical nature of this part of the flow. The mathematical advantage of this decomposition is, as in the steady case (Wundrow \& Goldstein 2001), that it allows for the removal of the explicit dependence of the three-dimensional part on the slow variable $\bar{y}$ (see below). The procedure may alternatively be viewed as a (purely) mathematical construction to achieve this purpose.

The governing equations for $\left(\hat{u}_{0}, \hat{v}_{0}, \hat{w}_{0}, \hat{p}_{1}\right)$, the three-dimensional part of the unsteady motion, follow from substituting (2.5) into the Navier-Stokes equations. Written in terms of the variable $y$, those equations involve $\bar{v}_{0}$. Similar to the case of the steady perturbations (Wundrow \& Goldstein 2001), the coupling with $\bar{v}_{0}$ (and the associated dependence on $\bar{y}$ ) can be removed by introducing the Prandtl transformation

$$
\hat{y}=y-\Re[\hat{\delta}(\bar{\xi}, \bar{t})],
$$

for a suitably chosen $\hat{\delta}$. Inserting (2.5) into the Navier-Stokes equations, and making use of (2.6) and (2.11), we obtain at leading order

$$
\begin{gathered}
\hat{v}_{0 y}+\hat{w}_{0 z}=0, \\
\mathcal{L}_{\mathcal{N}}\left(\begin{array}{c}
\hat{u}_{0} \\
\hat{v}_{0} \\
\hat{w}_{0}
\end{array}\right)=\left(\epsilon / k_{1}\right)\left(\begin{array}{c}
0 \\
-\hat{p}_{1 y} \\
-\hat{p}_{1 z}
\end{array}\right)+\sigma \nabla^{2}\left(\begin{array}{c}
\hat{u}_{0} \\
\hat{v}_{0} \\
\hat{w}_{0}
\end{array}\right),
\end{gathered}
$$

where we have put $\sigma=1 /\left(k_{1} R_{\Lambda}\right), \nabla^{2}$ is the Laplace operator in the $y-z$ plane, and the nonlinear differential operator

$$
\mathcal{L}_{\mathcal{N}}=\partial_{\bar{t}}+\partial_{\bar{x}}+\left(\epsilon / k_{1}\right)\left(\hat{v}_{0} \partial_{\hat{y}}+\hat{w}_{0} \partial_{z}\right)+\Re\left(-\hat{\delta}_{\bar{t}}-\hat{\delta}_{\bar{\xi}}+\left(\epsilon / k_{1}\right) \bar{v}_{0}\right) .
$$

The dependence on $\bar{v}_{0}$ can be removed by choosing $\hat{\delta}$ to make the last three terms vanish, leading to the equation

$$
\hat{\delta}_{\bar{\xi}}+\hat{\delta}_{\bar{t}}=\bar{\delta}_{\bar{\xi}}(\bar{\xi}, \bar{t})
$$

for $\hat{\delta}$ after (2.9) is used. The appropriate 'boundary condition' is

$$
\hat{\delta}(0, \bar{t})=0 \text { for all } \bar{t}>0,
$$

which corresponds to a vanishingly small displacement near the leading edge. Since the solution must be time periodic, the displacement thickness can be expressed as a Fourier series

$$
\bar{\delta}(\bar{x}, \bar{t})=\sum \bar{\delta}_{m}(\bar{x}) \mathrm{e}^{\mathrm{i} m(\bar{x}-\bar{t})},
$$

and the formal solution for $\hat{\delta}$ may accordingly be obtained as

$$
\hat{\delta}=\sum \hat{\delta}_{m}(\bar{\xi}) \mathrm{e}^{\mathrm{i} m(\bar{\xi}-\bar{t})} \quad \text { with } \quad \hat{\delta}_{m}=\bar{\delta}_{m}+\mathrm{i} m \int_{0}^{\bar{\xi}} \bar{\delta}_{m}(\bar{\xi}, \bar{t}) \mathrm{d} \bar{\xi} .
$$

The momentum equations $(2.13)$ indicate that the transverse velocities $\left(\hat{v}_{0}, \hat{w}_{0}\right)$ are 
decoupled from the streamwise velocity $\hat{u}_{0}$. The leading-order forcing of the free-stream disturbance on the boundary layer is exerted by $\left(\hat{v}_{0}, \hat{w}_{0}\right)$, to which the boundary-layer solution must match. The streamwise velocity so generated within the boundary layer is of $\mathcal{O}(1)$, while the direct forcing through $\hat{u}_{0}$ is of a higher-order effect. Matching $\hat{u}_{0}$ with the boundary-layer solution is necessary only when a solution with a higher accuracy of $\mathcal{O}\left(R_{\Lambda}^{-1}\right)$ is sought (cf. Ricco (2009)).

In view of the continuity equation (2.12), one may introduce the stream function $\hat{\psi}$, in terms of which the transverse momentum equations may be written as a transport equation for the longitudinal vorticity $\nabla^{2} \psi$,

$$
\left[\partial_{\bar{t}}+\partial_{\bar{x}}+\left(\epsilon / k_{1}\right)\left(\hat{\psi}_{z} \partial_{\hat{y}}-\hat{\psi}_{\hat{y}} \partial_{z}\right)\right] \nabla^{2} \hat{\psi}=\sigma \nabla^{4} \hat{\psi}
$$

and the Poisson equation for the pressure

$$
\nabla^{2} \hat{p}_{1}=-2\left(\hat{v}_{0 z} \hat{w}_{0 \hat{y}}+\hat{w}_{0 z}^{2}\right)=-2\left(\hat{\psi}_{\hat{y} \hat{y}} \hat{\psi}_{z z}+\hat{\psi}_{\hat{y} z}^{2}\right)
$$

It turns out that for a disturbance consisting of a pair of oblique modes, the nonlinear terms in (2.16) vanish identically, and the equation can be solved to obtain

$$
\hat{\psi}=2 c_{\infty} \sin \left(k_{3} z\right) \mathrm{e}^{-\sigma\left(k_{2}^{2}+k_{3}^{2}\right) \bar{x}+\mathrm{i} \bar{k}_{1}(\bar{x}-\bar{t})+\mathrm{i} k_{2} \hat{y}}+\text { c.c. },
$$

where $c_{\infty}$ is an arbitrary constant. It follows that

$$
\left(\hat{v}_{0}, \hat{w}_{0}\right)=\left(2 k_{3} \cos \left(k_{3} z\right),-2 \mathrm{i} k_{2} \sin \left(k_{3} z\right)\right) c_{\infty} \mathrm{e}^{-\sigma\left(k_{2}^{2}+k_{3}^{2}\right) \bar{x}+\mathrm{i}(\bar{x}-\bar{t})+\mathrm{i} k_{2} \hat{y}}+c . c .
$$

We take $c_{\infty}=-1 / k_{2}$ so that the normalized amplitude of the spanwise velocity in the free stream is unity, which fixes $\epsilon$. By inserting $\hat{\psi}$ into (2.17), the solution for the pressure is found as

$$
\hat{p}_{1}=2 k_{2}^{2}\left|c_{\infty}\right|^{2} \cos \left(2 k_{3} z\right) \mathrm{e}^{-2 \sigma\left(k_{2}^{2}+k_{3}^{2}\right) \bar{x}}-2 k_{3}^{2} c_{\infty}^{2} \mathrm{e}^{-2 \sigma\left(k_{2}^{2}+k_{3}^{2}\right) \bar{x}+2 \mathrm{i}(\bar{x}-\bar{t})+2 \mathrm{i} k_{2} \hat{y}}+\text { c.c. }
$$

To aid the matching with the solution in the boundary layer, we expand $\mathrm{e}^{-n \mathrm{i}\left(\bar{t}-k_{2} \hat{\delta}\right)}$ $(n=0,2)$ into Fourier series, i.e.

$$
\mathrm{e}^{-\mathrm{i} \bar{t}-\mathrm{i} k_{2} \hat{\delta}(\bar{x}, \bar{t})}=\sum_{m} \phi_{m}(\bar{x}) \mathrm{e}^{-\mathrm{i} m \bar{t}}, \quad \mathrm{e}^{-2 \mathrm{i} \bar{t}-2 \mathrm{i} k_{2} \hat{\delta}(\bar{x}, \bar{t})}=\sum_{m} \pi_{m}(\bar{x}) \mathrm{e}^{-\mathrm{i} m \bar{t}} .
$$

Then rewriting $\left(\hat{v}_{0}, \hat{w}_{0}, \hat{p}_{1}\right)$ in terms of $y$ by using $(2.11)$, we find that for $y=\mathcal{O}(1)$

$$
\left(\hat{v}_{0}, \hat{w}_{0}, \hat{p}_{1}\right)=\sum_{m, n}\left(v_{m, n}^{\dagger}, w_{m, n}^{\dagger}, p_{m, n}^{\dagger}\right) \mathrm{e}^{\mathrm{i} m(\bar{x}-\bar{t})+\mathrm{i} k_{3} n z},
$$

where

$$
\left.\begin{array}{rl}
v_{m, \pm 1}^{\dagger} & =k_{3} c_{\infty} \mathrm{e}^{-\sigma\left(k_{2}^{2}+k_{3}^{2}\right) \bar{x}}\left[\phi_{m} \mathrm{e}^{\mathrm{i}\left(\bar{x}+k_{2} y\right)}+\phi_{-m}^{*} \mathrm{e}^{-\mathrm{i}\left(\bar{x}+k_{2} y\right)}\right], \\
w_{m, \pm 1}^{\dagger} & =\mp k_{2} c_{\infty} \mathrm{e}^{-\sigma\left(k_{2}^{2}+k_{3}^{2}\right) \bar{x}}\left[\phi_{m} \mathrm{e}^{\mathrm{i}\left(\bar{x}+k_{2} y\right)}-\phi_{-m}^{*} \mathrm{e}^{-\mathrm{i}\left(\bar{x}+k_{2} y\right)}\right], \\
p_{0, \pm 2}^{\dagger} & =2 k_{2}^{2}\left|c_{\infty}\right|^{2} \mathrm{e}^{-2 \sigma\left(k_{2}^{2}+k_{3}^{2}\right) \bar{x}}, \\
p_{m, 0}^{\dagger} & =-2 k_{3}^{2} c_{\infty}^{2} \mathrm{e}^{-2 \sigma\left(k_{2}^{2}+k_{3}^{2}\right) \bar{x}}\left[\pi_{m} \mathrm{e}^{2 \mathrm{i}\left(\bar{x}+k_{2} y\right)}+\pi_{-m}^{*} \mathrm{e}^{-2 \mathrm{i}\left(\bar{x}+k_{2} y\right)}\right] ;
\end{array}\right\}
$$

all other components $v_{m, n}^{\dagger}=w_{m, n}^{\dagger}=0(n \neq \pm 1)$, and $p_{m, n}^{\dagger}=0(n \neq 0, \pm 2)$. Note that although the upstream disturbance is composed of only the temporal fundamental component, due to the displacement effect harmonics arise in fluctuations at the outer edge of the boundary layer as the disturbance evolves downstream. 


\subsection{The inner region: nonlinear unsteady streaks}

In the boundary layer, which corresponds to $\bar{x}=\mathcal{O}(1)$ and $y=\mathcal{O}(1)$, the flow can be decomposed as a sum of the Blasius boundary layer and the perturbation induced by the free-stream disturbance, namely

$$
\begin{gathered}
\{u, v, w, p\}=\left\{F^{\prime},\left(\frac{k_{1}}{2 \bar{x} R_{\Lambda}}\right)^{1 / 2}\left(\eta F^{\prime}-F\right), 0,-\frac{1}{2}\right\} \\
+r_{t}\left\{\bar{u}(\bar{x}, \eta, z, t),\left(\frac{2 \bar{x} k_{1}}{R_{\Lambda}}\right)^{1 / 2} \bar{v}(\bar{x}, \eta, z, t), \frac{k_{1}}{k_{3}} \bar{w}(\bar{x}, \eta, z, t), \frac{k_{1}}{R_{\Lambda}} \bar{p}(\bar{x}, \eta, z, t)\right\},
\end{gathered}
$$

where

$$
\eta=y\left(\frac{k_{1} R_{\Lambda}}{2 \bar{x}}\right)^{1 / 2} .
$$

The function $F(\eta)$ satisfies the Blasius equation

$$
F^{\prime \prime \prime}+F F^{\prime \prime}=0
$$

subject to the boundary conditions $F(0)=0, F^{\prime}(0)=0$ and $F^{\prime} \rightarrow 1$ as $\eta \rightarrow \infty$. For $\eta \gg 1$, the function $F \rightarrow \bar{\eta} \equiv \eta-\beta$, where $\beta \approx 1.2168$.

Note that the normal and spanwise velocities of streaks are still of small amplitude, but the streamwise velocity,

$$
U(y, z ; \bar{x}, \bar{t}) \equiv F^{\prime}+r_{t} \bar{u}(\bar{x}, \eta, z, t),
$$

is of $\mathcal{O}(1)$. The displacement effect, through which the viscous motion influences the inviscid outer region, is associated with the so-called transpiration velocity, i.e. the spanwise averaged velocity $v$ at the outer edge of the boundary layer. Integrating the continuity equation, $\partial U / \partial x+\partial v / \partial y+\partial w / \partial z=0$, first with respect to $z$ over a spanwise period $2 \pi / k_{3}$ and then with respect to $y$ from 0 to $\infty$, we find that

$$
\frac{k_{3}}{2 \pi} \int_{0}^{2 \pi / k_{3}} v(y, z ; \bar{x}, \bar{t}) \mathrm{d} z \rightarrow \frac{k_{3}}{2 \pi} \int_{0}^{2 \pi / k_{3}} \int_{0}^{\infty}\left(-U_{x}\right) \mathrm{d} y \mathrm{~d} z=k_{1} \bar{\delta}_{\bar{x}}(\bar{x}, \bar{t}) \text { as } y \rightarrow \infty,
$$

where $\bar{\delta}(\bar{x}, \bar{t})$ denotes the spanwise averaged displacement thickness, defined as

$$
\bar{\delta}(\bar{x}, \bar{t})=\frac{k_{3}}{2 \pi} \int_{0}^{2 \pi / k_{3}} \int_{0}^{\infty}(1-U(y, z, \bar{x}, \bar{t})) \mathrm{d} y \mathrm{~d} z .
$$

In the present nonlinear regime, the solution consists of all harmonics, and can be expressed as

$$
(\bar{u}, \bar{v}, \bar{w}, \bar{p})=\sum_{m, n=-\infty}^{+\infty}\left(\hat{u}_{m, n}(\bar{x}, \eta), \hat{v}_{m, n}(\bar{x}, \eta), \hat{w}_{m, n}(\bar{x}, \eta), \hat{p}_{m, n}(\bar{x}, \eta)\right) \mathrm{e}^{-\mathrm{i} m k_{1} t+\mathrm{i} n k_{3} z} .
$$

The reality of the physical quality requires

$$
\hat{q}_{-m,-n}=\left(\hat{q}_{m, n}\right)_{c c},
$$

where $\hat{q}_{m, n}$ stands for any of $\left(\hat{u}_{m, n}, \hat{v}_{m, n}, \hat{w}_{m, n}, \hat{p}_{m, n}\right)$, and the subscript $c c$ indicates the complex conjugate. The equations for the Fourier coefficients are obtained by inserting (2.23) and (2.29) into the nonlinear boundary-region equations, which are the rigorous asymptotic limit of the Navier-Stokes equations for $k_{1} \ll k_{3}$. The resulting equations are as follows. 
The Continuity equation

$$
\frac{\partial \hat{u}_{m, n}}{\partial \bar{x}}-\frac{\eta}{2 \bar{x}} \frac{\partial \hat{u}_{m, n}}{\partial \eta}+\frac{\partial \hat{v}_{m, n}}{\partial \eta}+n \mathrm{i} \hat{w}_{m, n}=0
$$

The $x$-Momentum equation

$$
\left(-i m-\frac{\eta F^{\prime \prime}}{2 \bar{x}}+n^{2} \kappa^{2}\right) \hat{u}_{m, n}+F^{\prime} \frac{\partial \hat{u}_{m, n}}{\partial \bar{x}}-\frac{F}{2 \bar{x}} \frac{\partial \hat{u}_{m, n}}{\partial \eta}-\frac{1}{2 \bar{x}} \frac{\partial^{2} \hat{u}_{m, n}}{\partial \eta^{2}}+F^{\prime \prime} \hat{v}_{m, n}=r_{t} \hat{f}_{m, n}
$$

The $y$-Momentum equation

$$
\begin{gathered}
\left(-i m+\frac{F^{\prime}}{2 \bar{x}}+\frac{\eta F^{\prime \prime}}{2 \bar{x}}+n^{2} \kappa^{2}\right) \hat{v}_{m, n}+\frac{1}{4 \bar{x}^{2}}\left(F-\eta F^{\prime}-\eta^{2} F^{\prime \prime}\right) \hat{u}_{m, n} \\
+F^{\prime} \frac{\partial \hat{v}_{m, n}}{\partial \bar{x}}-\frac{F}{2 \bar{x}} \frac{\partial \hat{v}_{m, n}}{\partial \eta}-\frac{1}{2 \bar{x}} \frac{\partial^{2} \hat{v}_{m, n}}{\partial \eta^{2}}+\frac{1}{2 \bar{x}} \frac{\partial \hat{p}_{m, n}}{\partial \eta}=r_{t} \hat{g}_{m, n}
\end{gathered}
$$

The $z$-Momentum equation

$$
\left(-i m+n^{2} \kappa^{2}\right) \hat{w}_{m, n}+F^{\prime} \frac{\partial \hat{w}_{m, n}}{\partial \bar{x}}-\frac{F}{2 \bar{x}} \frac{\partial \hat{w}_{m, n}}{\partial \eta}-\frac{1}{2 \bar{x}} \frac{\partial^{2} \hat{w}_{m, n}}{\partial \eta^{2}}+n \mathrm{i} \kappa^{2} \hat{p}_{m, n}=r_{t} \hat{h}_{m, n}
$$

where

$$
\kappa \equiv k_{3} /\left(k_{1} R_{\Lambda}\right)^{1 / 2}=\mathcal{O}(1)
$$

and the nonlinear terms $\hat{f}_{m, n}, \hat{g}_{m, n}$ and $\hat{h}_{m, n}$ are given by

$$
\begin{aligned}
& \hat{f}_{m, n}=\left[-\frac{\partial(\widehat{\bar{u}} \bar{u})}{\partial \bar{x}}+\frac{\eta}{2 \bar{x}} \frac{\partial \widehat{(\bar{u} \bar{u})}}{\partial \eta}-\frac{\partial \widehat{(\bar{u} \bar{v})}}{\partial \eta}-n i \widehat{\bar{u} \bar{w}}\right]_{m, n}, \\
& \hat{g}_{m, n}=\left[-\frac{1}{2 \bar{x}} \widehat{\widehat{u} \bar{v}}-\frac{\partial \widehat{(\bar{u} \bar{v})}}{\partial \bar{x}}+\frac{\eta}{2 \bar{x}} \frac{\partial \widehat{(\bar{u} \bar{v})}}{\partial \eta}-\frac{\partial \widehat{(\bar{v} \bar{v})}}{\partial \eta}-n i \widehat{\bar{v} \bar{w}}\right]_{m, n}, \\
& \left.\hat{h}_{m, n}=\left[-\frac{\partial(\widehat{\bar{u} \bar{w}})}{\partial \bar{x}}+\frac{\eta}{2 \bar{x}} \frac{\partial \widehat{(\bar{u} \bar{w})}}{\partial \eta}-\frac{\partial(\overline{\bar{v} \bar{w}})}{\partial \eta}-n i \widehat{\bar{w} \bar{w}}\right]_{m, n}, \quad\right]
\end{aligned}
$$

with the ${ }^{\wedge}$ symbol denoting Fourier transform.

\subsection{Initial and outer boundary conditions}

While the induced perturbation within the boundary layer acquires an $\mathcal{O}(1)$ streamwise velocity when $\bar{x}=\mathcal{O}(1)$, all three velocity components are of small amplitude near the leading edge so that perturbation is essentially linear there. As is explained in the appendix, the upstream conditions derived by LWG remain valid. These can be expressed as

$$
\begin{gathered}
\hat{u}_{1, \pm 1} \rightarrow q_{ \pm}(2 \bar{x})\left(U_{0}+(2 \bar{x})^{1 / 2} U_{1}\right) \\
\hat{v}_{1, \pm 1} \rightarrow q_{ \pm}\left\{V_{0}+(2 \bar{x})^{1 / 2} V_{1}+\frac{\mathrm{i}}{\left(\kappa_{2}-\mathrm{i}|\kappa|\right)(2 \bar{x})^{1 / 2}}\left(\mathrm{e}^{\mathrm{i} \kappa_{2}(2 \bar{x})^{1 / 2} \bar{\eta}-\left(\kappa^{2}+\kappa_{2}^{2}\right) \bar{x}}-\mathrm{e}^{-|\kappa|(2 \bar{x})^{1 / 2} \bar{\eta}}\right)\right. \\
-\left(\frac{3}{4} \beta-\frac{1}{2} g_{1}|\kappa|(2 \bar{x})^{1 / 2}\right) \mathrm{e}^{-|\kappa|(2 \bar{x})^{1 / 2} \bar{\eta}}+\bar{\eta}+\frac{3}{4} \beta \\
\left.+(2 \bar{x})^{1 / 2}\left[-\frac{\mathrm{i}}{2}\left(\kappa_{2}+\mathrm{i}|\kappa|\right)\left(\bar{\eta}^{2}+1\right)+\frac{3}{4} \beta|\kappa| \bar{\eta}+\frac{1}{2}|\kappa| g_{1}\right]\right\} \\
\hat{w}_{1, \pm 1} \rightarrow \mp \mathrm{i} q_{ \pm}\left\{W_{0}+(2 \bar{x})^{1 / 2} W_{1}+\frac{1}{\kappa_{2}-\mathrm{i}|\kappa|}\left(\kappa_{2} \mathrm{e}^{\mathrm{i} \kappa_{2}(2 \bar{x})^{1 / 2} \bar{\eta}-\left(\kappa^{2}+\kappa_{2}^{2}\right) \bar{x}}-\mathrm{i}|\kappa| \mathrm{e}^{-|\kappa|(2 \bar{x})^{1 / 2} \bar{\eta}}\right)\right.
\end{gathered}
$$




$$
\left.-\frac{3}{4} \beta|\kappa|(2 \bar{x})^{1 / 2} \mathrm{e}^{-|\kappa|(2 \bar{x})^{1 / 2} \bar{\eta}}-1-(2 \bar{x})^{1 / 2}\left[\mathrm{i}\left(\kappa_{2}+\mathrm{i}|\kappa|\right) \bar{\eta}-\frac{3}{4} \beta|\kappa|\right]\right\},
$$

as $\bar{x} \rightarrow 0$, where

$$
q_{ \pm}= \pm\left(\mathrm{i} \kappa^{2} / k_{3}\right)\left(\hat{u}_{3, \pm}^{\infty} \pm \mathrm{i} \hat{u}_{2, \pm}^{\infty}\right), \quad \kappa_{2}=k_{2} / \sqrt{k_{1} R_{\Lambda}}
$$

the constant $g_{1}$ is given in the equation (B 15) on page 200 in LWG, and $U_{k}, V_{k}$ and $W_{k}$ $(k=0,1)$ are found by solving the system (B 1)-(B 8) in LWG. The transversely decaying term in $(2.38)-(2.39), \mathrm{e}^{-|\kappa|(2 \bar{x})^{1 / 2} \bar{\eta}}$, represents the reflected disturbance by the wall. The upstream conditions (2.37)-(2.39) prove to be adequate for starting calculations, as our numerical results will show.

The solution within the boundary layer must match the outer solution (2.21). Thus for $\bar{x}=\mathcal{O}(1)$, we require that

$$
\left(\hat{u}_{m, n}, \hat{v}_{m, n}, \hat{w}_{m, n}, \hat{p}_{m, n}\right) \rightarrow\left(0,(2 \bar{x} / \sigma)^{-\frac{1}{2}} v_{m, n}^{\dagger},\left(k_{3} \sigma\right) w_{m, n}^{\dagger},\left(\epsilon / k_{1}\right) p_{m, n}^{\dagger}\right)
$$

for $n \neq 0$ as $\eta \rightarrow \infty$, where $\left(v_{m, n}^{\dagger}, w_{m, n}^{\dagger}, p_{m, n}^{\dagger}\right)$ on the right-hand side are given by (2.22).

The boundary-region equations (2.31)-(2.34), along with the upstream conditions (2.37)(2.39) and the outer boundary condition (2.40), and further supplemented by (2.28), (2.15) and (2.26), describe the entrainment of free-stream disturbances and the subsequent development of the induced streaks.

\section{Numerical procedures}

The boundary-region equations (2.31)-(2.34) are parabolic in the $\bar{x}$-direction and hence can be solved by a marching procedure in $\bar{x}$. The equations are discretised by a secondorder finite-difference scheme which is backward in $\bar{x}$ and central in $\eta$. The resulting block tri-diagonal system at each $\bar{x}$ is solved using a standard block-elimination algorithm. The pressure component is computed on a grid staggered in the $\eta$ direction with respect to the grid for the velocity field so as to avoid pressure decoupling. No boundary condition for the pressure fluctuation is required at the wall; its value is calculated a posteriori by solving the $z$-momentum equation at $\eta=0$. The equations for components with $n=0$ require special attention as the pressure $\hat{p}_{m, 0}$ appears only in the $y$-momentum equation. The three velocity components are thus computed by only solving the continuity, $x$ - and $z$-momentum equations, and no far-field condition is required. The wall-normal derivative of the pressure can be obtained a posteriori from the $y$-momentum equation.

The outer boundary conditions (2.40) in the upstream limit $\bar{x} \ll 1$ are consistent with the initial conditions (2.37)-(2.39) when $\kappa(2 \bar{x})^{1 / 2} \eta \gg 1$; both represent the oncoming disturbance since $\mathrm{e}^{-|\kappa|(2 \bar{x})^{1 / 2} \bar{\eta}}$ (i.e. the reflected disturbance) is negligible. The condition for overlapping (i.e. $|\kappa|(2 \bar{x})^{1 / 2} \eta \gg 1$ ) implies that $\eta$ has to be large if the Dirichlet outer boundary conditions (2.40) is employed for very small $\bar{x}$. In order to avoid this, the mixed boundary conditions (5.28)-(5.31) of LWG, which are consistent with (2.40) but accommodate the transverse decay of the reflected disturbance, are used to march the BREs for a short distance, typically to $\bar{x}=0.04-0.07$, over which the perturbation is linear. Farther downstream, the boundary condition is switched to (2.40). Careful numerical checks have been performed to ensure that the result is independent of the location of the switching, and that the normal and spanwise velocity components match smoothly to the values specified by $(2.40)$.

The Hermitian property, (2.30), can be used to reduce the size of the matrix. Fourier modes with negative indices $m$ are evaluated by using (2.30) so that only half of the Fourier modes need to be computed. The nonlinear terms are evaluated by using the 

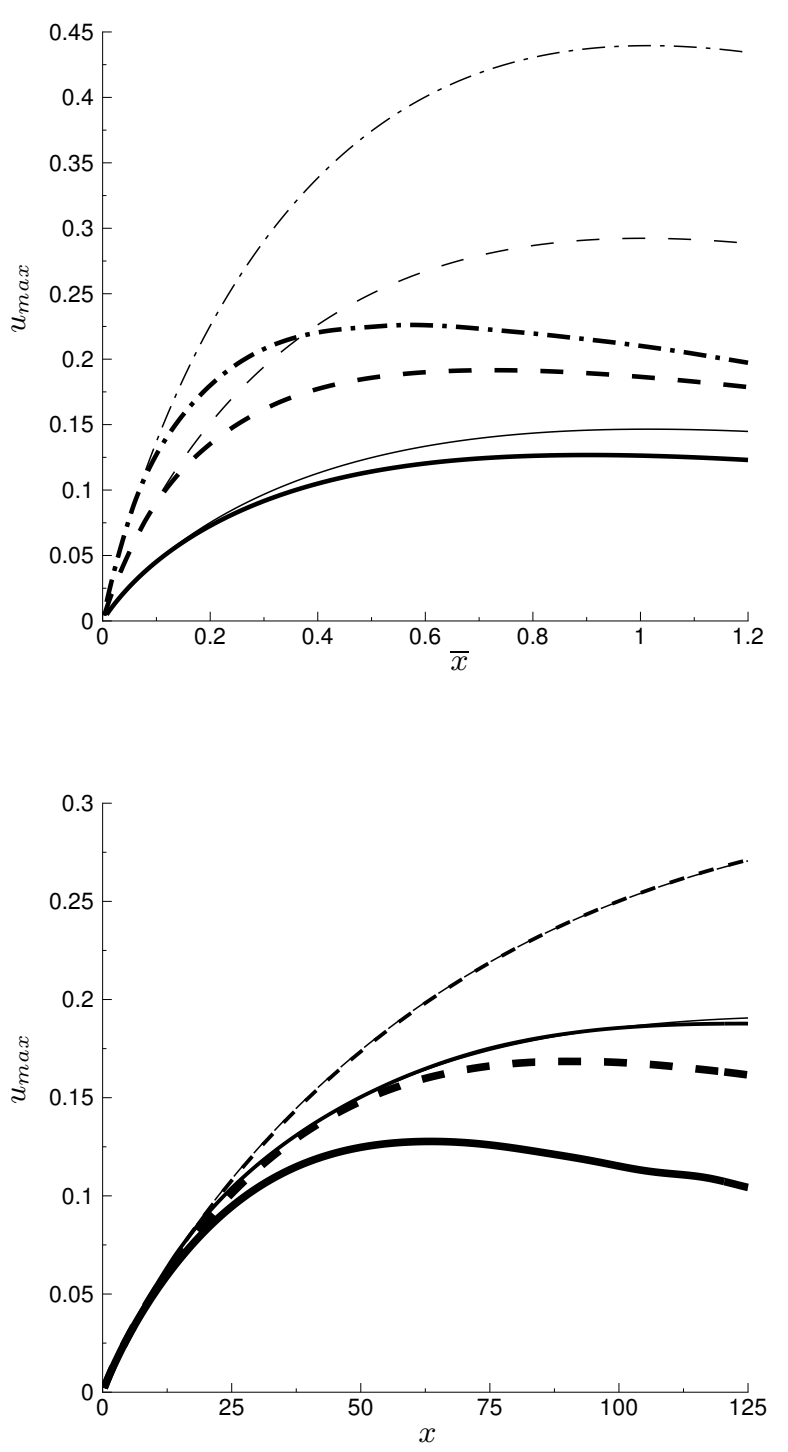

FIgURE 2. Linear and nonlinear development of $u_{\max }$, the maximum r.m.s. of the streamwise velocity of the streak. Top: $u_{\max }$ v.s. $\bar{x}$ for a fixed $k_{1}=0.005$, and different $\epsilon=0.005$ (solid lines), 0.01 (dashed lines), 0.015 (dash-dotted lines). Thin/thick curves denote the linear/nonlinear solutions. Bottom: $u_{\max }$ v.s. $x$ at a fixed $r_{t}=4\left(R_{\Lambda}=400, \epsilon=0.01\right)$, and different $k_{1}=0.005$ (thin line), 0.01 and 0.05 (thick lines). Dashed/solid lines indicate linearised/nonlinear solutions.

pseudo-spectral method, that is the velocities in spectral space are transformed back to physical space in order to carry out multiplications. The products are subsequently Fourier transformed back again to spectral space. A second-order predictor-corrector scheme is employed for handling the nonlinear effect. In the predictor, the nonlinear terms are treated explicitly: they are approximated by using the velocity field at the three previous $\bar{x}$ locations. The resulting linear algebraic system is solved to evaluate the velocity and pressure field. This predicted solution is used in a correction step to 


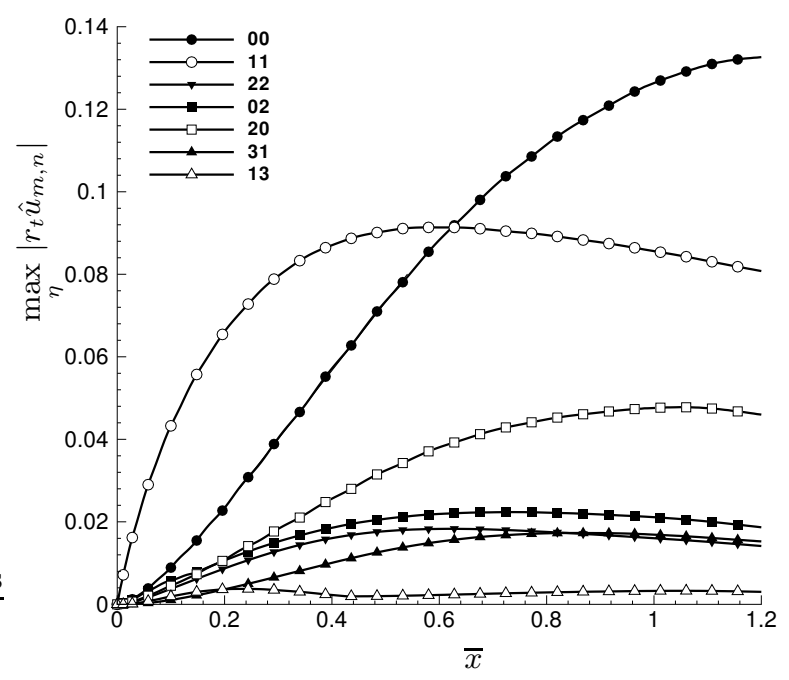

Figure 3. Development of the fundamental mode max $\left|r_{t} \hat{u}_{11}\right|$, and nonlinearly generated harmonic components $\max \left|r_{t} \hat{u}_{m, n}\right|$. The parameters are $k_{1}=0.005, R_{\Lambda}=400$ and $\epsilon=0.01$.

recompute the nonlinear terms. The iteration is repeated until the difference between the consecutive estimates is smaller than a specified tolerance. The aliasing error is eliminated by following the so-called 3/2-rule, which prevents the spurious energy cascade from the unresolved high-frequency modes into the resolved low-frequency ones (Kim et al. 1987). Such a de-aliasing procedure was found to be essential for the stability of the numerical results when nonlinearity becomes significant. Use of $N_{t}=N_{z}=17$ Fourier modes is sufficient to capture the nonlinear effect. Resolution checks show that truncated modes have an order-of-amplitude of about $10^{-10}$, and thus do not significantly influence the flow dynamics. The magnitude of the error remains constant throughout the downstream evolution of the flow. The domain extends to $\eta=30$, and 1000 grid points are used in this direction.

\section{Results for the nonlinear development of streaks}

The parameters characterizing the free-stream disturbance are taken to be as follows: $\hat{u}_{1,2, \pm}^{\infty}=1.0, \hat{u}_{3, \pm}^{\infty}=\mp 1.0$, which leads to $k_{1}+k_{2}-1=0$. Unless otherwise indicated, we take $k_{1}=0.005, R_{\Lambda}=400(\kappa=0.707)$ and $\epsilon=0.01\left(r_{t}=4\right)$, for which the FST intensity, defined as the r.m.s. of the free-stream streamwise velocity, is $T u=2 \sqrt{2} \epsilon=2.8 \%$. This case is chosen because it is representative of typical low-speed wind-tunnel experiments, where, for example, $U_{\infty}=5 \mathrm{~m} / \mathrm{s}$, the spanwise wavelength is $\lambda_{z}^{*} \approx 8 \mathrm{~mm}$, energetic disturbances in the boundary layer are found to be in the frequency band $f^{*}<10 \mathrm{~Hz}$, and the free-stream turbulence intensity is $T u=1-3 \%$; see for example Westin et al. (1994); Matsubara \& Alfredsson (2001); Fransson et al. (2005).

Due to the nonlinear effect, the streak signature consists of all harmonics. Its overall intensity may be measured by the r.m.s., $u_{r m s}$, defined as (see Pope (2000), page 687)

$$
u_{r m s} \equiv r_{t}\left[\sum_{m, n}\left|\hat{u}_{m, n}\right|^{2}\right]^{1 / 2}, m \neq 0 .
$$



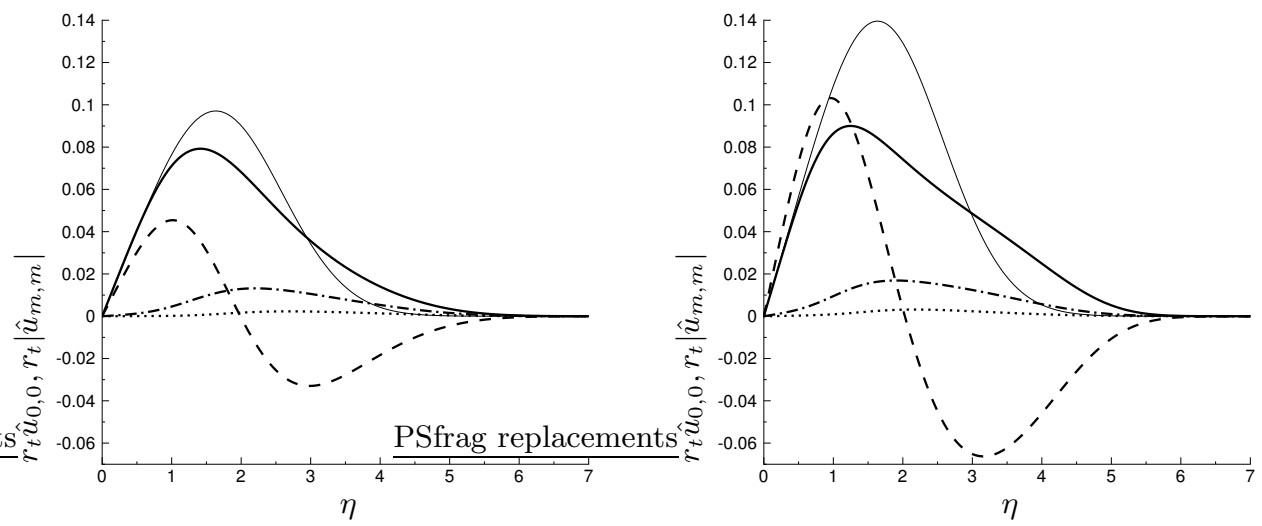

Figure 4. Profiles of the streamwise velocities of the mean-flow distortion and harmonics $u_{m, m}$ ( $m=1,2,3)$ at $\bar{x}=0.3$ (left) and $\bar{x}=0.7$ (right). Dashed line: $r_{t} \hat{u}_{0,0}$; solid line: $r_{t}\left|\hat{u}_{1,1}\right|$ (thin: linear solution, thick: nonlinear solution); dashed-dotted line: $r_{t}\left|\hat{u}_{2,2}\right|$, and dotted line: $r_{t}\left|\hat{u}_{3,3}\right|$.

Figure 2 (top) shows the downstream development of $u_{\max }$, the maximum of $u_{r m s}$ at each $\bar{x}$ defined as

$$
u_{\max } \equiv \max _{\eta} u_{r m s}
$$

for three different values of $\epsilon$. Sufficiently upstream, the streak signature is weak so that the linear and nonlinear solutions overlap. Nonlinearity gradually asserts its influence as the disturbance amplifies. Compared with the corresponding linear solution, the nonlinear disturbance grows more slowly, attenuates appreciably earlier, and its peak amplitude is also reduced, indicating that nonlinearity plays a stabilizing role. It may therefore be inferred that the amplitude of the streaks would be significantly over-predicted by linearised theory. The stabilizing effect becomes more pronounced as $\epsilon$ increases. For instance, the nonlinear responses for $\epsilon=0.01$ and $\epsilon=0.015$ differ by just $20 \%$ despite the $50 \%$ difference in the amplitude of the free-stream disturbance. Figure 2 (bottom) indicates that the stabilizing effect is also enhanced for smaller values of $k_{1}$ up to $k_{1}=0.01$. For $k_{1}$ below 0.01 , which are typical of disturbances causing bypass transition, the evolution of $u_{\max }$ is almost indistinguishable from each other, suggesting that nonlinearity stabilizes uniformly all components with sufficiently long wavelengths.

A stabilizing effect of nonlinearity was noted in previous studies in related but different contexts. For example, Leib et al. (1999b) found that nonlinearity tends to inhibit the boundary-layer response to a steady perturbation in the free stream. A similar effect has also been reported by Zuccher et al. (2006) in their study of steady optimal perturbations in the Blasius boundary layer.

The nonlinear self-interaction of each fundamental mode generates a mean-flow distortion as well as higher harmonics $\hat{u}_{m, \pm m}$. Figure 3 shows the development of $\max _{\eta}\left|r_{t} \hat{u}_{m, n}\right|$, the maximum amplitude of these components. Near the leading edge, all harmonics and the mean-flow distortion have much smaller amplitudes than that of the fundamental, consistent with the linear nature of the disturbance in this region. The harmonics remain of smaller amplitude for $\bar{x}=\mathcal{O}(1)$, but the mean-flow distortion $\hat{u}_{00}$ acquires a magnitude appreciably greater than the fundamental. A strong mean-flow distortion leads to formation of a 'backward jet', an issue to be discussed later. Figure 4 displays the streamwise velocity profiles of the fundamental mode $(1,1)$, the mean-flow distortion 

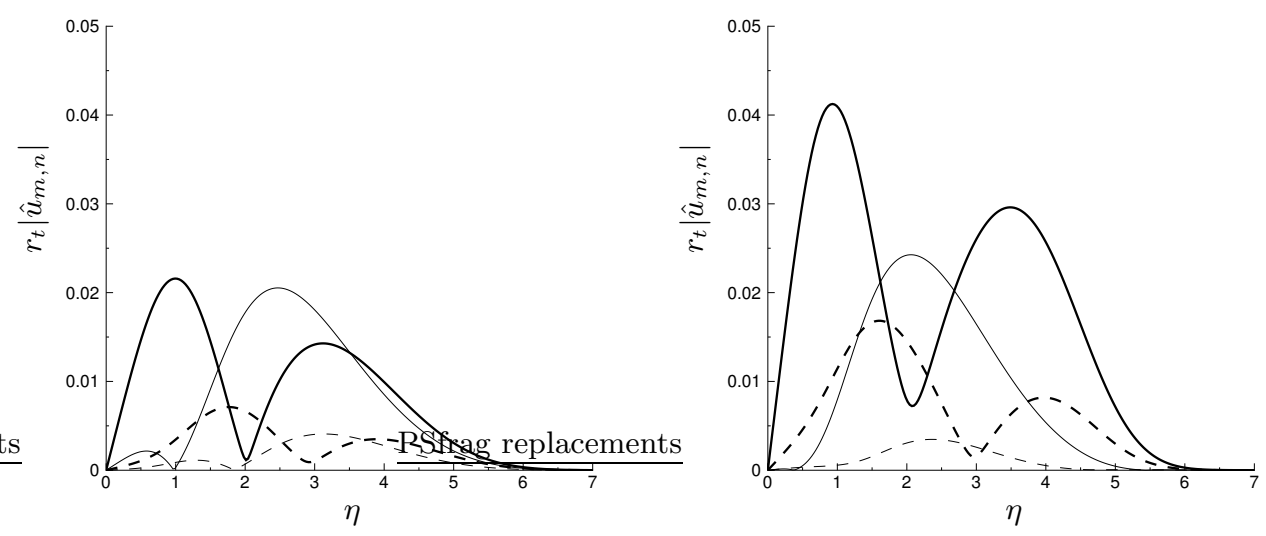

FiguRE 5. Profiles of the streamwise velocities of the harmonics $u_{m, n}(m \neq n)$ at $\bar{x}=0.3$ (left) and at $\bar{x}=0.7$ (right). Thin solid line: $r_{t}\left|\hat{u}_{0,2}\right|$; thick solid line: $r_{t}\left|\hat{u}_{2,0}\right|$; thin dashed line: $r_{t}\left|\hat{u}_{1,3}\right|$, thick dashed line: $r_{t}\left|\hat{u}_{3,1}\right|$.

$(0,0)$, and the second and third harmonics $(2,2)$ and $(3,3)$, at $\bar{x}=0.3$ and 0.7 . The result is presented only for modes with $n \geq 0$ since modes $(m, n)$ and $(m,-n)$ have the same amplitude for the free-stream disturbance of the assumed form. The magnitude of the higher harmonics decreases so quickly that the third harmonic $(m=3)$ makes an almost negligible contribution to the overall disturbance energetics. The mean-flow distortion undergoes considerable amplification as it evolves downstream, while the magnitudes of the harmonics vary very little in this range of $\bar{x}$. Note that the linear (thin solid line) and the nonlinear (thick solid line) profiles of $r_{t}\left|\hat{u}_{1,1}\right|$ overlap for $\eta<0.5$, suggesting that viscous effects dominate over the Reynolds stress in this near wall region. The peak of the disturbance is located closer to the wall in the nonlinear case than in the linear case. Nonlinearity attenuates the disturbance in the core, but enhances fluctuations in the outer portion $(\eta>3)$ of the boundary layer.

The mutual interaction between $(1,1)$ and $(1,-1)$ generates additional harmonic components $(2,0)$ and $(0,2)$. Their profiles, displayed in figure 5 as thick and thin solid lines respectively, have comparable amplitudes. The $(0,2)$ mode features a peak in the core of the boundary layer at $\eta \approx 2$, while the $(2,0)$ mode has two peaks at $\eta \approx 1$ and 3.5. The mean-flow distortion $(0,0)$ has a magnitude about four times larger than that of the harmonics $(2,0)$ and $(2,2)$, but the spanwise-dependent mean-flow component $(0,2)$ (i.e. steady streak) does not acquire an even larger amplitude as one might anticipate on the basis of wave-wave interactions (cf. Goldstein \& Choi (1989), Wu et al. (1993), Hall $\&$ Smith (1991)). This is because the streamwise wavelength of the fundamental modes $(1, \pm 1)$ is long enough to be comparable with the length scale over which their amplitude evolves, which means that the harmonics and steady streaks are hardly distinguishable in the sense that they all have the same streamwise length scale. As a result, they have comparable magnitudes.

The components generated at third order, $\hat{u}_{3,1}$ and $\hat{u}_{1,3}$, are displayed by dashed lines in figure 5 ; the amplitude of $\hat{u}_{3,1}$ is larger than that of $\hat{u}_{1,3}$. Similarly to $\hat{u}_{2,0}$, the profile of $\hat{u}_{3,1}$ also exhibits two peaks, but the respective peak positions are located farther from the wall, at $\eta \approx 2$ and 4 respectively.

We now examine the steady streaks $u_{s t r}$, i.e. the steady spanwise modulation of the streamwise velocity generated by nonlinear interactions. Mathematically, it corresponds 


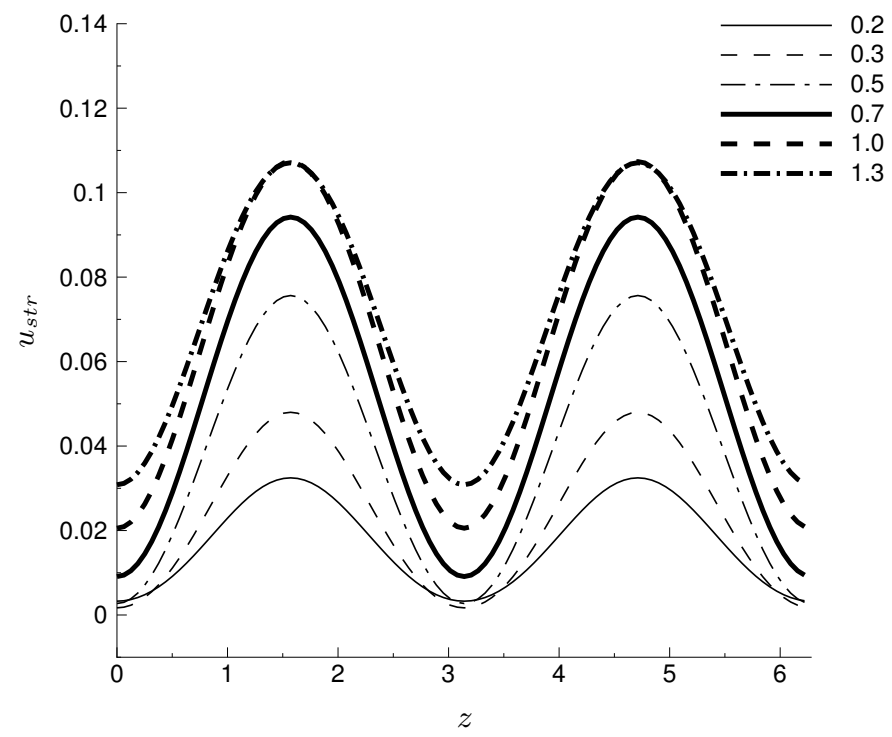

FiguRE 6. Amplitude and spanwise distribution of steady streaks $u_{\text {str }}$ (see (4.3)) at $\eta=1.64$ at different $\bar{x}$.

to the sum of Fourier components $(0, \pm n)$, i.e.

$$
u_{s t r} \equiv r_{t} \sum_{n} \hat{u}_{0, n} \mathrm{e}^{\mathrm{i} n k_{3} z}
$$

Steady streaks are superimposed onto the Blasius boundary layer to give the total steady streamwise velocity

$$
U_{M}(\bar{x}, \eta, z)=F^{\prime}(\eta)+u_{s t r}(\bar{x}, \eta, z) .
$$

Theoretical results concerning steady streaks are worth documenting because $U_{M}$ (and $\left.u_{s t r}\right)$ can readily be acquired in the laboratory by taking the time average of point-wise measurements (and subtracting out the Blasius profile).

We first consider $u_{\text {str }}$ at $\eta=1.64$, which roughly corresponds to the location of the maximum. The spanwise distributions of $u_{\text {str }}$ at different $\bar{x}$ are shown in figure 6 . Steady streaks exhibit a pattern of spanwise alternating 'valleys' and 'peaks', which remain aligned in the streamwise direction. Their intensity increases as the flow evolves downstream. The steepening spanwise gradient is caused by the accumulated effect of the low-speed fluid near the wall being lifted up while the high-speed fluid in the outer portion is brought down towards to the wall. Figure 7 shows the contours of $u_{\text {str }}$ in the $y-z$ plane at different streamwise locations. The outer region of the boundary layer (i.e. $\eta \approx 3$ ) features a lower velocity with respect to the Blasius value, while the opposite occurs near the wall. The outer low-velocity region becomes thinner in the $\eta$-direction, an indication of an increasing normal gradient.

The time- and $z$-averaged streamwise velocity,

$$
\bar{U}_{M}=U_{M}(\bar{x}, \eta)=F^{\prime}(\eta)+r_{t} \hat{u}_{0,0}(\bar{x}, \eta)
$$

is now studied. Figure 8 shows the profiles of the mean-flow distortion $r_{t} \hat{u}_{0,0}$ at different $\bar{x}$. The amplitude of $r_{t} \hat{u}_{0,0}$ grows downstream. The induced mean velocity is positive near 
(a)

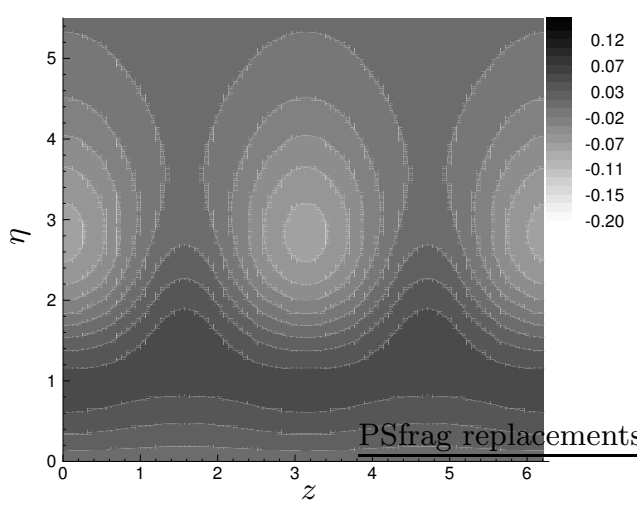

(c)

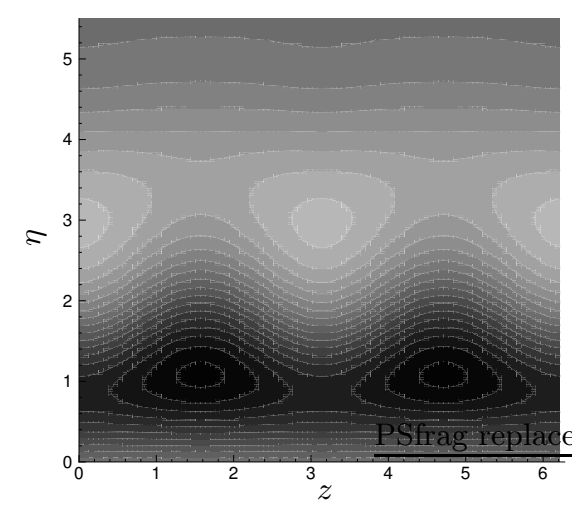

(b)

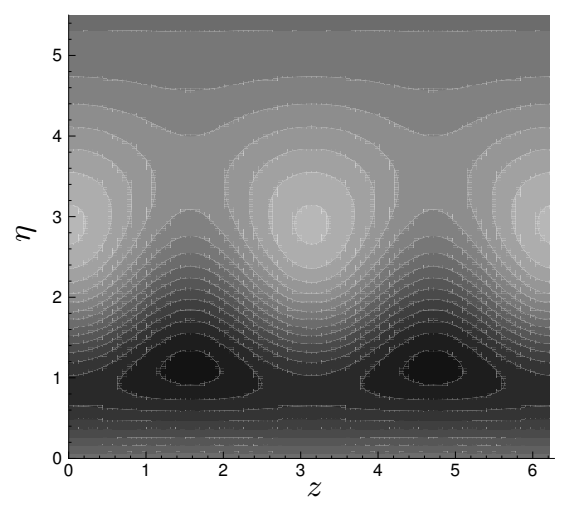

(d)

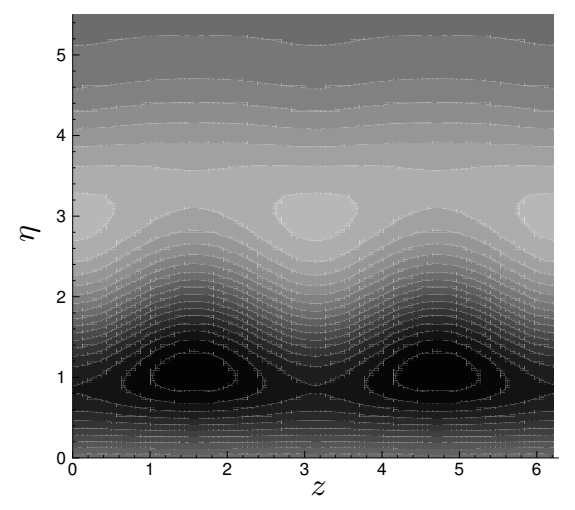

FIGURE 7. Contour of steady streamwise velocity streaks in $\eta-z$ plane at $\bar{x}=0.3(\mathrm{a}), 0.7(\mathrm{~b})$, $1.0(\mathrm{c})$ and $1.2(\mathrm{~d})$.

the wall and negative in the outer region, indicating respectively an increase of the mean wall-shear stress with respect to the Blasius value and a 'backward jet' forming at the edge of the boundary layer (Jacobs \& Durbin 2001). Interestingly, the profile at different $\bar{x}$ crosses zero almost always at $\eta \approx 2$.

An attempt is now made to compare the theoretical predictions with the wind-tunnel experimental data of Matsubara \& Alfredsson (2001) for the case of $U_{\infty}=12 \mathrm{~m} / \mathrm{s}, T u=$ $1.5 \%$. In these experiments, the boundary layer is perturbed by a continuous spectrum of turbulent disturbances, while in our formulation the free-stream disturbance is synthesized by a pair of convected gusts. A precise quantitative comparison is not possible at this stage, but the theory may be expected to capture salient qualitative features if the wavelengths and frequency of the free-stream disturbance are chosen to correspond to those of the dominant component in the FST in the experiments. The characteristic spanwise wavelength can be estimated as the minimum of the autocorrelation function $R_{u u}$ of the streamwise velocity perturbation along $z$. From figure 7 of Matsubara \& Alfredsson (2001), one finds that $\lambda_{z}^{*}=0.008 \mathrm{~m}$, a value also consistent with flow visualization images. The representative frequency, $f^{*}=5 \mathrm{~Hz}$, can be extracted from their figure $9 \mathrm{~b}$, where spectra of disturbances are shown. The gust intensity can be found through the value of $T u$, i.e. $\epsilon=0.01 T u /(2 \sqrt{2})=0.0053$. Table 1 presents the estimated parameters 


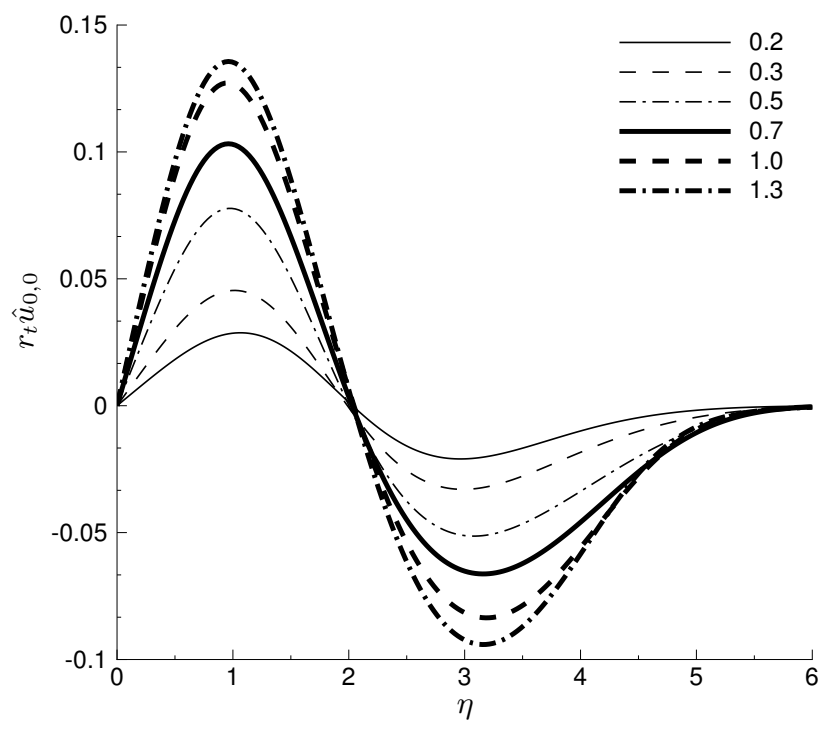

FiguRE 8. Profiles of $r_{t} \hat{u}_{0,0}$ at different $\bar{x}$.
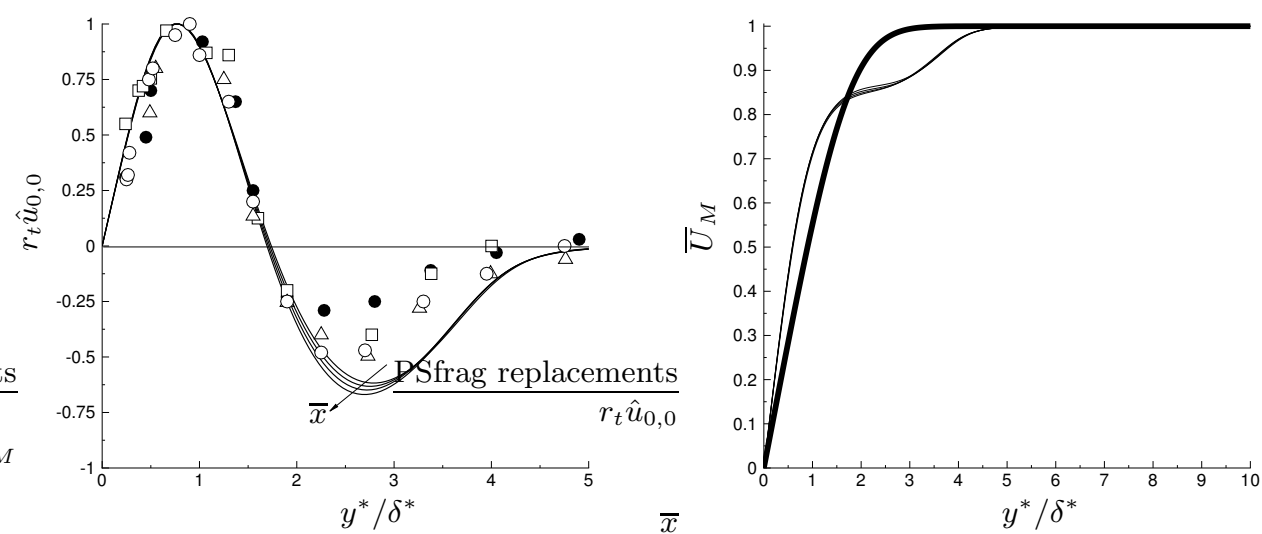

Figure 9. Left: Profiles of $r_{t} \hat{u}_{0,0}$ rescaled by the maximum along $y^{*} / \delta^{*}$ and comparison with the experimental data of Matsubara \& Alfredsson (2001) at $x^{*}=0.7 \mathrm{~m}$ (filled circles), $0.8 \mathrm{~m}$ (squares), $0.9 \mathrm{~m}$ (triangle) and $1 \mathrm{~m}$ (open circles). Right: Profiles of the mean streamwise velocity $\bar{U}_{M}$ at different $\bar{x}$ (thin lines) and of the Blasius profile (thick line).

pertinent to the experiments of Matsubara \& Alfredsson (2001). The fact that the scaled spanwise wavenumber $\kappa=\mathcal{O}(1)$ and that $\lambda_{z}^{*}$ is comparable with the boundary-layer thickness highlights the importance of accounting for the spanwise viscous diffusivity, while the disturbance Reynolds number $r_{t}=\mathcal{O}(1)$ confirms the relevance of nonlinear effects. It is also found that $\lambda_{x}^{*} \gg \lambda_{z}^{*}$, as required by the asymptotic formulation.

Computations were performed using the parameters given in table 1 . The predicted profiles of the mean-flow distortion $r_{t} \hat{u}_{0,0}\left(y^{*} / \delta^{*}\right)$ at four different streamwise locations are shown in the left graph of figure 9 , where $\delta^{*}$ is the displacement thickness. The 

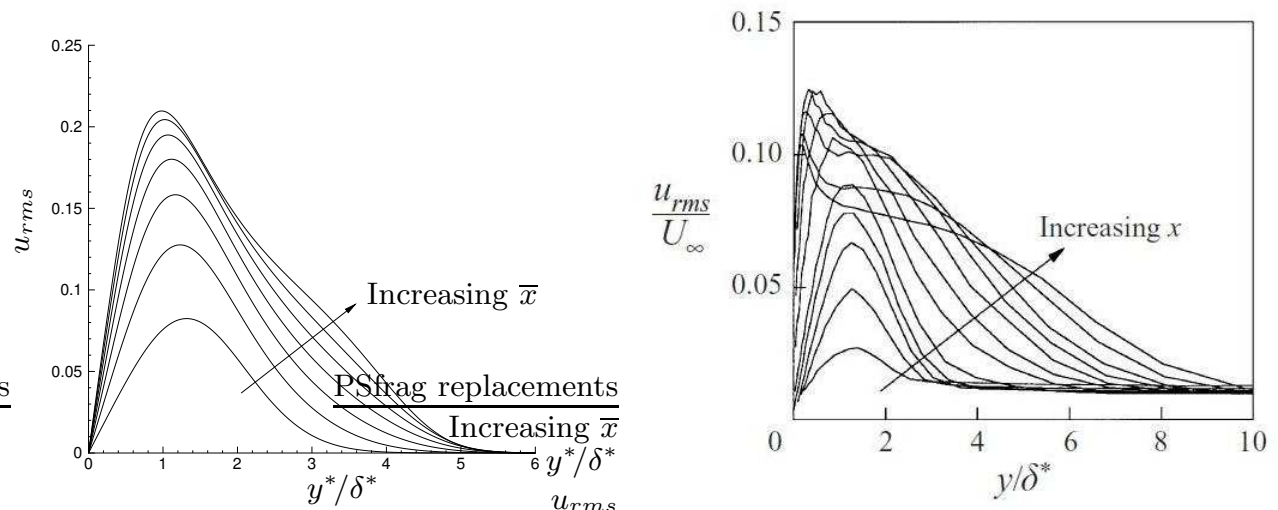

FiguRE 10. Profiles of $u_{r m s}$, the r.m.s. of the streamwise velocity. Left: Predicted profiles at $\bar{x}=0.1,0.2,0.3,0.4,0.6,0.7$. Right: Measurement of figure 2c of Matsubara \& Alfredsson (2001).

\begin{tabular}{ccccccc}
$\lambda_{x}^{*}(\mathrm{~m})$ & $\lambda_{z}^{*}(\mathrm{~m})$ & $f(\mathrm{~Hz})$ & $k_{1}$ & $\kappa$ & $R_{\Lambda}$ & $r_{t}$ \\
\hline 2.4 & 0.008 & 5 & 0.0033 & 0.555 & 974.4 & 5.17
\end{tabular}

TABLE 1. Estimated flow parameters pertinent to the experimental data shown in figure 2 of Matsubara \& Alfredsson (2001).

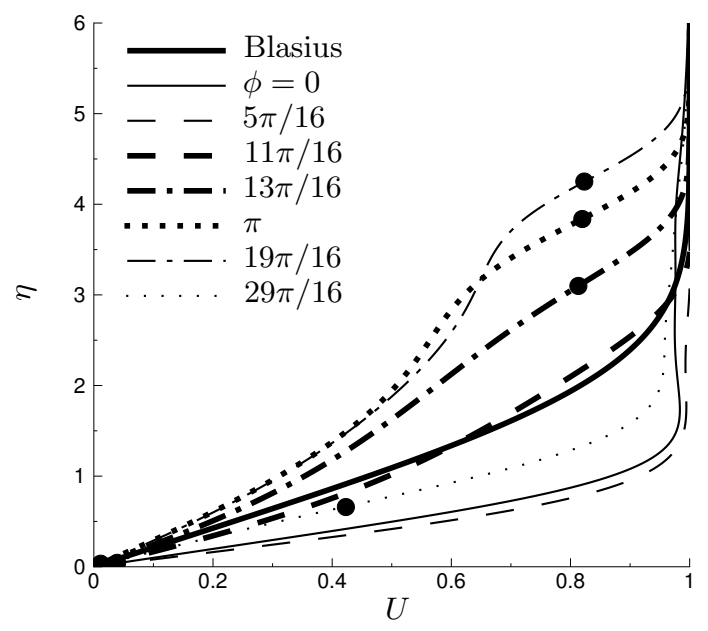

Figure 11. Profiles of the instantaneous streamwise velocity $U(\eta, z ; \bar{x}, \bar{t})($ see $(2.26))$ at $z=0$ and $\bar{x}=1.2$ in different phases. Inflection points are marked by $\bullet$

normalized profiles match the wind-tunnel data (symbols) quite well, in particular for $y^{*} / \delta^{*}<2$. The shape of the profile in this range of $y^{*} / \delta^{*}$ is independent of the streamwise location. An appreciable but rather small change of shape is noted for $y^{*} / \delta^{*}>2$. The minimum is over predicted, but its wall-normal location $\left(y^{*} / \delta^{*} \approx 3\right)$ is captured accurately, and the slight decrease of the minimum with $x^{*}$ is consistent with the ex- 
perimental data. The discrepancies in the outer portion of the boundary layer may be attributed to the simplified representation of the free-stream disturbance. Better agreement may be expected if the latter is modelled more realistically, e.g. by a stochastic Fourier series having the same spectral property of the FST in experiments.

The predicted profiles of the mean flow $\bar{U}_{M}$ at different $\bar{x}$ are displayed in the right graph of figure 9 . The profiles at $x^{*}=0.7,0.8,0.9,1 \mathrm{~m}(\bar{x}=1.83,2.09,2.36,2.62$, respectively - thin lines) collapse well over the whole boundary layer, suggesting that nonlinear effects have saturated at these sufficiently far downstream locations. The increase of the wall-shear stress over the Blasius solution (the thick line) agrees with the experimental observation.

Figure 10 (left) shows how the profile of $u_{r m s}$ of the disturbance evolves downstream. Near the leading edge, the boundary-layer disturbance is of small amplitude so that its profile agrees with the theoretical linear solution (LWG), $\eta F^{\prime \prime}$, the shape of which resembles the eigen solution of Luchini (1996). In the downstream region where the nonlinear effects become relevant, the fundamental mode in the main bulk of the boundary layer grows at a slower rate, while the disturbance in the outer region becomes more energetic. The peak position moves towards the wall. Both trends are in good qualitative agreement with the experimentally measured $u_{r m s}$ profiles, shown in figure 2c of Matsubara \& Alfredsson (2001). The latter is reproduced in the right hand graph of figure 10; the profiles at the last three locations should be ignored as the flow has entered the fully turbulent regime, in which $u_{r m s}$ is caused by small-scale fluctuations rather than by streaks.

The instantaneous streamwise velocity profiles at $\bar{x}=0.7$ and $z=0$ in different phases

$$
\phi \equiv k_{1} t
$$

are shown in figure 11. The boundary-layer response in the near-wall region is characterized by forward and backward fluctuations, but with the former being prevalent. At the outer edge of the boundary layer $(2.7<\eta<5)$, perturbations are persistently in the negative streamwise direction, forming a region of velocity deficit, or a 'backward jet'. These results agree with previous findings by DNS (Jacobs \& Durbin 2001).

The instantaneous streamwise velocity profile is found to become inflectional in the normal direction during certain phases or time windows of the oscillations as is shown in figure 11, where the inflection points $\eta_{s}$ satisfying the Fjørtoft's criterion, (Drazin \& Reid 2004)

$$
U^{\prime \prime}\left(\eta_{s}\right)=0 \quad \text { and } \quad U^{\prime \prime}\left[U-U\left(\eta_{s}\right)\right]<0,
$$

are marked. In flows which are steady and spanwise-uniform, the presence of such points is a necessary condition for inviscid instability. The base flow of the present study, i.e. the boundary layer perturbed by streaks, is unsteady and spanwise dependent. Nevertheless, appearance of inflection points may be taken as an precursor of inviscid secondary instability on the basis of the following observations. Firstly, the base flow may be treated as being quasi-steady because the characteristic time scale of inviscid modes is much shorter than that of underlying streaks. Secondly, a connection between an inflectional profile and inviscid instability was suggested by stability calculations of steady spanwisedependent flows (Malik et al. 1999). Furthermore, it has been shown mathematically that an inflectional profile in the region where $\delta^{*} \ll \Lambda$ indeed becomes inviscidly unstable (Wu \& Luo 2003; Wu \& Choudhari 2003).

Figure 12 shows contours of the instantaneous streamwise velocity at $\bar{x}=0.7$ and 1.2. In each case, three phases of time modulation are chosen. The flow field exhibits a mushroom-like shape, which is associated with the near-wall low-speed fluid moving toward the outer portion of the boundary layer, while at the location of half a spanwise 
wavelength away, the high-speed fluid in the outer part is brought down towards the wall. This spanwise alternating pattern is also observed in boundary layers perturbed by steady free-stream disturbances (Wundrow \& Goldstein 2001), but the spanwise concentration of vorticity in the present unsteady case is much less pronounced. This is probably because the up- and down-wash motions leading to concentration reverse their directions during a cycle of modulation.

\section{Streak instability}

The nonlinear calculations predict a distorted velocity profile $U(y, z ; \bar{x}, \bar{t})$, which may in certain phases possess inflection points in the streamwise and spanwise directions, and hence may become inviscidly unstable. Since $U$ varies with $\bar{x}$ and $\bar{t}$ slowly, the dependence on these two variables can be treated as being parametric when the short wavelength (of order $\delta^{*}$ ) and the high frequency (of order $U_{\infty} / \delta^{*}$ ) instability is considered. For the secondary instability mode, the perturbation, pressure $\tilde{p}$ say, takes the form

$$
\tilde{p}=\widehat{p}(y, z ; \bar{x}, \bar{t}) \mathrm{e}^{\mathrm{i}(\alpha x-\omega t)} .
$$

Owing to the dependence of the base flow on both $y$ and $z, \widehat{p}$ is governed by a partial differential equation (Hall \& Horseman 1991)

$$
\left[(U-\omega / \alpha)\left(\partial_{y}^{2}+\partial_{z}^{2}-\alpha^{2}\right)-2 U_{y} \partial_{y}-2 U_{z} \partial_{z}\right] \widehat{p}=0 .
$$

This equation together with appropriate boundary conditions leads to a bi-global instability problem (Theofilis 2003).

Since $U$ has been obtained as a function of $\eta$, we rewrite (5.2) in terms of $\eta$. For spanwise-periodic streaks,

$$
U(\eta, z ; \bar{x}, \bar{t})=U\left(\eta, z+2 \pi / k_{3} ; \bar{x}, \bar{t}\right) \equiv \sum_{m} \bar{U}_{m}(\eta ; \bar{x}, \bar{t}) \mathrm{e}^{\mathrm{i} m k_{3} z},
$$

Floquet theory can be used to express $\widehat{p}$ as

$$
\widehat{p}(y, z ; \bar{x}, \bar{t})=\mathrm{e}^{\mathrm{i} q k_{3} z} \sum_{n} \widehat{p}_{n}(\eta ; \bar{x}, \bar{t}) \mathrm{e}^{\mathrm{i} n k_{3} z},
$$

where $0 \leq q \leq \frac{1}{2}$ with $q=0$ and $q=\frac{1}{2}$ representing the fundamental and subharmonic resonance respectively. Substitution into (5.2) yields a system of ordinary differential equations of infinite dimensions for $\widehat{p}_{n}$,

$$
\begin{aligned}
\sum_{m}\{ & \left.\bar{U}_{n-m} \widehat{p}_{m}^{\prime \prime}-2 \bar{U}_{n-m}^{\prime} \widehat{p}_{m}^{\prime}-\left[(m+q)^{2} \hat{k}_{3}^{2}+\hat{\alpha}^{2}+2(n-m)(m+q) \hat{k}_{3}^{2}\right] \bar{U}_{n-m} \widehat{p}_{m}\right\} \\
& =(\hat{\omega} / \hat{\alpha})\left[\widehat{p}_{n}^{\prime \prime}-\left((m+q)^{2} \hat{k}_{3}^{2}+\hat{\alpha}^{2}\right) \widehat{p}_{n}\right]
\end{aligned}
$$

where a prime denotes differentiation with respect to $\eta$, and

$$
\left(\hat{\alpha}, \hat{k}_{3}, \hat{\omega}\right)=\left(\alpha, k_{3}, \omega\right)\left[2 \bar{x} /\left(k_{1} R_{\Lambda}\right)\right]^{\frac{1}{2}} .
$$

The no-penetration condition and the requirement that the mode decays at the infinity impose the boundary conditions,

$$
\widehat{p}_{n, \eta}(0)=0, \quad \widehat{p}_{n} \rightarrow 0 \quad \text { as } \quad \eta \rightarrow \infty
$$

on (5.4). The infinite system (5.4)-(5.5) is to be truncated and discretized using an appropriate discretisation scheme. 
Temporal instability of steady spanwise-periodic streaks has been studied previously (Andersson et al. 2001). The present investigation differs from the earlier work in that streaks are induced by physically realizable free-stream disturbance rather than represented by an optimal disturbance. This difference is crucial because only by considering FST-induced streaks can the effect of FST on instability and transition be quantified. Another distinction is that in addition to temporal instability, spatial instability, which is potentially more relevant, will be considered.

The infinite domain for $\eta$ is mapped to the finite interval $[-1,1]$, and $\widehat{p}_{m}$ is expanded as a series,

$$
\widehat{p}_{m}=\sum a_{m n} T_{n}(\zeta)
$$

of Chebyshev polynomials $T_{n}$, where $\zeta$ denotes the new variable after the mapping. Projection of (5.4) at the collocation points leads to an algebraic system of the form, $\mathbf{A}(\alpha) \mathbf{a}=(\hat{\omega} / \hat{\alpha}) \mathbf{B a}$, where $\mathbf{A}$ and $\mathbf{B}$ are matrices. This generalized eigenvalue problem is solved by an approach involving two steps. For the temporal instability formulation, in which $\hat{\alpha}$ is real and $c=\hat{\omega} / \hat{\alpha}=c_{r}+\mathrm{i} c_{i}$ is complex, the eigenvalue problem is linear and the QZ method is used to compute all the eigenvalues for a given $\hat{\alpha}$. The focus is on the eigenvalue $c$ with the largest imaginary part $c_{i}$ (i.e. the most unstable mode), while the remaining ones are discarded because their imaginary parts are at least one order-of-magnitude smaller. The value of $c$ given by the QZ method is taken as a first approximation and is further refined in the second step, which consists of a Gaussian elimination procedure and the Muller iteration to force the determinant $|\mathbf{A}-c \mathbf{B}|=0$. The QZ approximation is used as the first guess to initiate the iteration. When the convergence is achieved, the corresponding eigenfunction is computed.

For the spatial instability formulation, the eigenvalue problem is nonlinear since the complex wavenumber $\hat{\alpha}$ has to be found for a given real frequency $\hat{\omega}$. The QZ method is not directly applicable even though the Muller method may be implemented provided that a good initial guess for $\hat{\alpha}$ can be specified. A 'parametric continuation' procedure is adopted. The temporal instability is extended to allow for a complex $\hat{\alpha}=\hat{\alpha}_{r}+\mathrm{i} \hat{\alpha}_{i}$, and is solved using the Muller method to find $\hat{\omega}=\hat{\omega}_{r}+\mathrm{i} \hat{\omega}_{i}$. Then $\hat{\alpha}_{r}$ is fixed but $-\hat{\alpha}_{i}$ is gradually increased from zero until $\hat{\omega}_{i}=0$, by which stage a temporal mode is continued into a spatial mode.

Stability calculations of nonlinear streaks were performed for the case $R_{\Lambda}=400$, $k_{1}=0.005$ and $\epsilon=0.01$. Eight Fourier terms and 256 Chebyshev polynomials were used. Doubling Fourier terms to 16 in the Muller method yields no appreciable difference. Results will be presented for the fundamental modes $(q=0)$ since they are found to be more unstable than the subharmonic $\left(q=\frac{1}{2}\right)$ or detuned modes $\left(0<q<\frac{1}{2}\right)$.

Figure 13 shows the instability characteristics at $\bar{x}=0.7$, where streaks reach their maximum amplitude and the spanwise wavelength of streaks is found to be about $6 \delta^{*}$. Calculations of the growth rate at different instants indicate that, within each cycle of streak oscillation, instability occurs in two time windows, $\pi / 2<\phi<\pi$ and $3 \pi / 2<\phi<$ $2 \pi$. Each instability episode lasts about a quarter of the cycle. The temporal growth rates corresponding to the three instants, $\phi=25 \pi / 16,15 \pi / 8,31 \pi / 16$, are shown in figure 13a. The band of unstable modes have wavenumbers $\hat{\alpha}<1.2$ with the largest growth rate being attained for $\hat{\alpha} \approx 0.5$, or $\alpha \approx 0.6$, suggesting that the most unstable mode has a streamwise wavelength about 1.7 times larger than the spanwise wavelength of streaks. The phase speed and group velocity are shown in figure 13b. The phase speed appears to be only weakly dependent on the frequency, and remains in the range of $0.75-0.82 U_{\infty}$. The group velocity is close to the phase speed with the maximum difference being about just $10 \%$. The predicted phase and group velocities are in agreement with the value $(c \approx 0.8)$ 


\begin{tabular}{lcrrrrrr} 
& $R_{\delta^{*}}$ & $F$ & $\alpha_{r}^{\dagger}\left(\lambda_{s}^{*}\right)$ & $c_{r}$ & $c_{g}$ & $-\alpha_{i}^{\dagger}$ & $\Delta t^{*} U_{\infty} / \delta^{*}$ \\
\hline & & & & & & & \\
Theory $(\bar{x}=0.7)$ & 407 & 1243 & $0.63\left(10 \delta^{*}\right)$ & 0.79 & 0.75 & 0.049 & 270 \\
Theory $(\bar{x}=1.2)$ & 533 & 1186 & $0.80\left(7.7 \delta^{*}\right)$ & 0.77 & 0.72 & 0.074 & 240 \\
Experiment & $588-715$ & & $\left(16 \delta^{*}\right)$ & 0.8 & & 0.01 & $(50)$
\end{tabular}

TABLE 2. Instability characteristics of the most unstable modes and comparison with the experimental data of Mans et al. (2007).

estimated from experimental observations (Mans et al. 2007). The propagation speed does not appear to be sensitive to the profile and amplitude of streaks. Similar values were obtained from numerical studies of impulse propagation in a boundary boundary perturbed by an optimal disturbance (Brandt et al. 2003; Schlatter et al. 2008).

The spatial growth rates of unstable modes, calculated by solving the spatial eigenvalue problem as described above, are displayed in figure 13c. Spatial growth rates may alternatively be obtained using Gaster transformation (Gaster 1962),

$$
\hat{\alpha}_{i}=-\hat{\omega}_{i} / c_{g}
$$

The result is compared with the directly computed $-\alpha_{i}$. There is hardly any difference to graphic precision, which is rather surprising because the relation (5.6) is expected to hold only when $\hat{\omega}_{i}$ is very small.

Figure 14 displays the (normalized) eigenfunctions $\left|\widehat{p}_{m}\right|(m=1,2,3,4,5)$ of the most unstable modes in the phases $\phi=25 \pi / 16,15 \pi / 8$. An interesting feature is that the first harmonic $\widehat{p}_{1}$ always has a much larger amplitude than that of the other components. At each instant, the most unstable temporal and spatial modes resume the identical shape.

An investigation of the stability of streaks is also carried out at a location further downstream, i.e. at $\bar{x}=1.2$. The results are shown in figures 15 and 16 . The overall characteristics are similar to those at $\bar{x}=0.7$. At $\bar{x}=1.2$, the amplitude of streaks is somewhat smaller than that at $\bar{x}=0.7$ (see figure 2 (top)), and yet the band of unstable modes appears broader and their growth rates are larger, i.e. streaks are more unstable despite having a smaller amplitude. This suggests that the magnitude of streaks is not the only relevant parameter determining the instability; their spatial structure is also important. Again, the spatial growth rates obtained by using (5.6) are remarkably accurate. Only in the most unstable phase (curve 3 in figure 15) can one detect a very small (but negligible) difference from the directly calculated $-\hat{\alpha}_{i}$.

The streamwise velocity $\widehat{u}$ of the instability mode is related to the pressure $\widehat{p}$ via the relation

$$
\widehat{u}(y, z)=-\left(\widehat{p}_{y y}+\widehat{p}_{z z}+\alpha^{2} \widehat{p}\right) /\left(2 \alpha^{2}(\bar{U}-c)\right) .
$$

Contours of $\widehat{u}(y, z)$ are shown in figure 17 , in which contours of the instantaneous streamwise velocity of streaks are included. The mode resides in the outer portion of the boundary layer and on the 'shoulders' of streaks. This feature is broadly similar to that exhibited by sinuous modes supported by a steady optimal disturbance (Andersson et al. 2001). It is worth noting that $\widehat{u}$ mostly concentrates in the vicinity of the critical level $\eta_{c}(z)$ where $\bar{U}\left(\eta_{c}, z\right)=c_{r}$.

In order to aid the comparison with previous results and experiments, we convert the characteristics of the most unstable modes at $\bar{x}=0.7$ and 1.2 to quantities normalized in the same way as in most of the experimental literature, and the streamwise location 
to the Reynolds number,

$$
R_{\delta^{*}}=U_{\infty} \delta^{*} / \nu=1.2168\left(2 \bar{x} R_{\Lambda} / k_{1}\right)^{1 / 2}
$$

based on the local displacement thickness $\delta^{*}$. The result is displayed in table 2 . The position $\bar{x}=0.7$ corresponds to $R_{\delta^{*}}=407$, which is smaller than the critical Reynolds number $R_{\delta^{*}} \approx 520$ for the T-S instability (Jordinson 1970). The streak instability considered here takes place in the region where T-S waves are all damped, and it is therefore likely to be the inherent mechanism of bypass transition. The frequency of the most unstable mode, measured by

$$
F \equiv\left(2 \pi f^{*} \nu / U_{\infty}^{2}\right) \times 10^{6}=\left(\omega / R_{\Lambda}\right) \times 10^{6},
$$

is $F \approx 1186$, where $f^{*}$ is the dimensional frequency in Hertz. In contrast, unstable T-S waves are in the frequency band $F<400$, and those which cause transition are actually of much lower frequency with $F \approx 10^{2}$ because T-S modes with $F>200$ soon reach the upper branch and decay (Fasel \& Konzelman 1990; Kachanov 1994). The $\alpha^{\dagger}$ in the table is the wavenumber normalized by $\delta^{*}$, and it is related to $\hat{\alpha}$ by $\alpha^{\dagger}=\beta \hat{\alpha}$. The most unstable mode has $\alpha_{r}^{\dagger} \approx 0.63$, which gives a streamwise wavelength $\lambda_{s}^{*} \approx 10 \delta^{*}$. The predicted growth rate of $-\hat{\alpha}_{i} \approx 0.04$ for the most unstable mode corresponds to $-\alpha_{i}^{\dagger} \approx 0.049$, which is one order-of-magnitude greater than that of T-S waves; the latter is about $\mathcal{O}\left(10^{-3}\right)$ in the major unstable zone (Jordinson 1970; Fasel \& Konzelman 1990). The location $\bar{x}=1.2$ corresponds to $R_{\delta^{*}} \approx 533$, which is just about in the unstable region of T-S waves. The most unstable inviscid mode has more or less the same character as that at $\bar{x}=0.7$, except that its growth rate is considerably larger.

As a comparison, the experimental data of Mans et al. (2007) is also shown in the table 2. Using the combined PIV-visualization technique in a water channel, Mans et al. (2007) detected sinuous wavy motions of streaks in the region covering $1.17 \times 10^{5}<R e_{x}<$ $1.73 \times 10^{5}$, or $588<R_{\delta^{*}}<715$, where $R e_{x}$ is the Reynolds number based on the distance to the leading edge. Although two-dimensional T-S modes are unstable in this range of the Reynolds number, highly three-dimensional ones are damped, and therefore the possibility of the observed wavy motions being oblique T-S waves can be ruled out. Mans et al. (2007) reported a propagation velocity of $0.8 U_{\infty}$, which is in close agreement with our prediction. The wavelength was estimated to be about $40 \delta_{300}^{*}$ (and $\delta_{300}^{*}=2.4 \mathrm{~mm}$ for $U_{\infty}=0.125 \mathrm{~m} / \mathrm{s}$ ), which corresponds, according to Schlatter et al. $(2008)$, to $9-16 \delta^{*}$, but our estimate gives $16-20 \delta^{*}$ depending on the location of observation. The value of $16 \delta^{*}$ entered in table 2 is probably closer to the lower end, but is still about twice as large as the typical value suggested by the theory. The spatial growth rate $\left(-\alpha_{i}^{\dagger}\right)$ is the quantity that is most difficult to measure. Nevertheless, Mans et al. (2007) was able to extract an average value of 0.01 , which is smaller than the theoretical estimate. This level of discrepancy is not surprising because the characteristics of free-stream disturbances, on which both the wavelength and growth rate depend, must be rather different. On the other hand, the scattering in the raw data is so large that the experimental values should probably be viewed as merely an indication of the orders of magnitude. The present theory uses a rather simplistic representation of FST, but appears to predict the right orders of magnitude.

Our calculations indicate that streak instability occurs within about a quarter of cycle, i.e. $\Delta \phi \approx \pi / 2$. The corresponding time window $\Delta t^{*}$, normalised by $\delta^{*} / U_{\infty}$, is 270 at $\bar{x}=0.7$ and 240 at $\bar{x}=1.2$. Such an intermittent nature of the instability was suggested by the experimental observation of Mans et al. (2007), where the time lapse from the onset of sinuous oscillations to the breakdown into small-scale motions was found to be about 50. That this duration is a fraction of the time window for instability seems reasonable 
because unstable modes must first undergo amplification before becoming observable, and must have acquired sufficiently large magnitude to cause transition before the unstable phase ends.

A full validation of the theoretical prediction requires careful experiments in which the free-stream vortical disturbances are introduced in a controlled and repeatable manner. Controlled two-dimensional gusts were successfully generated in the study of boundarylayer receptivity (Dietz 1999). It would be interesting to use a similar technique to generate a pair of oblique gusts as assumed in the theory. We hope that the present work would spur such an experiment, in which case the results shown in table 2 and in figures 13 and 15 might provide a useful guide.

\section{Summary and conclusions}

As a crucial step towards understanding and predicting bypass transition, we investigated the influence of unsteady free-stream disturbance, modelled by convected gusts, on the Blasius boundary layer. Attention is focused on long-wavelength components, which are known to penetrate into the boundary layer to generate streamwise elongated streaks. For sufficiently weak disturbances, an initial-boundary-value problem, consisting of the linearised BREs and appropriate upstream and far-field boundary conditions, has been formulated by LWG to describe the entrainment of the disturbance and the evolution of streaks. That formulation has in the present study been generalized to the case where the free-stream disturbance is strong enough to generate streaks with $\mathcal{O}(1)$ streamwise velocity thereby fundamentally altering the stability characteristics of the flow. In the region where the local boundary-layer thickness becomes comparable with the spanwise wavelength scale, the streaks are governed by the nonlinear BREs. The disturbance in the far field becomes nonlinear too and is simultaneously influenced by the displacement effect produced by the viscous motion in the boundary layer.

The resulting nonlinear initial-boundary-value problem is solved numerically for the special case where the convected gust consists of a pair of oblique modes with the same frequency but opposite spanwise wavenumbers. Nonlinearity is found to have a stabilizing effect in that it inhibits the amplification of streaks, and causes the fluctuation to attenuate earlier. Nonlinear interactions within the boundary layer generate fluctuations at harmonic frequencies as well as an appreciable mean-flow distortion. The profile of the latter exhibits a velocity deficit at the edge of the boundary layer so that the distorted mean flow features a 'backward jet' as was observed in DNS (Jacobs \& Durbin 2001). The profiles of the mean-flow distortion and the r.m.s of the fluctuation are in reasonably good agreement with the experimental measurements of Matsubara \& Alfredsson (2001).

During each cycle, there exist time windows in which the instantaneous velocity profile becomes inflectional in the wall-normal direction, suggesting that the streaky boundary layer may be inviscidly unstable. This is confirmed by both temporal and spatial secondary instability analyses. Calculations for a disturbance level $T u=2.8 \%$ indicate that intermittent but robust instability occurs in two time windows, each lasting about one quarter of the cycle. The dominant modes are of sinuous type and have the same spanwise wavelength as that of streaks. Their characteristic wavelength, growth rate, phase and group velocities are comparable with available experimental data. It may be pointed out that this is the first stability calculation of nonlinear unsteady streaks induced by a realizable (though still idealized) free-stream disturbance. It is also the first time that spatial growth rates of secondary instability have been calculated directly by solving the nonlinear eigenvalue problem. Our result indicates that the Gaster transformation, frequently used to convert temporal growth rates to spatial ones, is remarkably accurate. 
In the present work, streaks are assumed to be spanwise periodic so that the secondary instability was formulated by using Floquet theory. Experiments indicate that streak breakdown occurs sporadically in space, suggesting that local instability of isolated streaks operates. This instability was found to arise in the region where $\delta^{*} \ll \Lambda$ (Wu \& Choudhari 2003; Wu \& Luo 2003). It would be interesting to investigate further the instability of nonlinear localized streaks in the generic case where $\delta^{*}=\mathcal{O}(\Lambda)$.

The present work focused on the relatively high level of FST for which robust inviscid instability occurs upstream of the lower branch of the much weaker viscous T-S instability. FST of moderate level is incapable of causing inviscid instability, but may significantly alter the growth rates of T-S modes. The asymptotic analysis in the limit $\delta^{*} \ll \Lambda$ shows that streaks enhance the amplification of upper-branch T-S waves, and convert them into long-wavelength inviscidly unstable modes as the FST intensity increases (Wu \& Choudhari 2003). However, as mentioned in the introduction, the effect of streaks and the associated mean-flow distortion on the T-S instability across its entire spectrum (including the lower-branch regime) remains controversial. Further insights may be gained by including viscosity in the stability formulation and solving the resultant eigenvalue problem.

Finally, it should be emphasised that the present formulation can be extended to account for a continuum of low-frequency components in FST, which are relevant disturbances causing bypass transition. The entire process of initiation and development of streaks can be described appropriately by BREs. Unlike DNS, BREs can be solved efficiently thereby making extensive parametric study possible. Because of these features, the present theoretical framework may form a basis for developing an efficient and physicsbased method to correlate the variation of transition location with $T u$ and to predict the critical threshold turbulence level delineating bypass and T-S transition. For instance, a systematical secondary-instability analysis may be performed to establish a quantitative relationship between the onset location $x_{s}$ of the instability and $T u$, i.e. $x_{s}=x_{s}(T u)$. The critical threshold $T u_{c}$ may be estimated as the value of $T u$ for which $x_{s}$ coincides with the critical Reynolds number for the T-S instability. Since streak instability leads to rapid amplification, its onset location $x_{s}$ may be taken as a first approximation for the bypass transition point. An improved correlation could be made based on the accumulated amplification of the streak instability.

\section{Acknowledgements}

This work is supported by the UK EPSRC (grant no. EP/F045093/1) and the Royal Society of London under the International Joint Project Scheme. Part of the research work was conducted by PR during the 2004 Summer Research Program of the Center for Turbulence Research (CTR) at Stanford University. He would like to acknowledge the hospitality and financial support of the CTR, as well as helpful discussions with Professors Paul Durbin, Sanjiva Lele, and Dr Tamer Zaki (now at Imperial College London). The authors would like to thanks the referees for their helpful comments and suggestions. 


\section{Appendix A. Matching with the upstream linear solution}

In this appendix, we show that the nonlinear solution in the downstream region where $\bar{x}=\mathcal{O}(1)$ matches to the upstream linear stage in the overlapping region $R_{\Lambda}^{-1} \ll \bar{x} \ll 1$. Similar to the case of steady disturbance considered by Wundrow \& Goldstein (2001), the boundary layer in this limit splits into two regions corresponding to $\eta=\mathcal{O}(1)$ and $y=\mathcal{O}(1)$. These and the outer region where $y \gg 1$ are now considered.

In the outer region $y \gg 1$, observe first that as $\bar{x} \rightarrow 0$,

$$
\bar{\delta} \rightarrow \beta(2 \bar{x})^{1 / 2} / \sqrt{k_{1} R_{\Lambda}},
$$

since the streamwise velocity of streaks is expected to be of small amplitude near the leading edge. It follows from (2.11) and (2.14) that $\hat{\delta}=\bar{\delta}$, and

$$
\hat{y} \rightarrow y^{(0)} / \sqrt{k_{1} R_{\Lambda}},
$$

so that $\hat{v}_{0}$ and $\hat{w}_{0}(2.18)$ match the oncoming disturbance $(2.1)$.

In the region $\eta=\mathcal{O}(1)$, we may seek power-series solution (as in the linear case considered by LWG),

$$
\left(\hat{u}_{m, n}, \hat{v}_{m, n} \cdot \hat{w}_{m, n}, \hat{p}_{m, n}\right)=\sum_{k=0}^{\infty}(2 \bar{x})^{k / 2}\left((2 \bar{x}) U_{m, n}^{(k)}, V_{m, n}^{(k)}, W_{m, n}^{(k)}, P_{m, n}^{(k)} /(2 \bar{x})^{1 / 2}\right) .
$$

Substitution of the expansion into (2.31)-(2.34) shows that $\left(U_{m, n}^{(k)}, V_{m, n}^{(k)}, W_{m, n}^{(k)}, P_{m, n}^{(k)}\right)$ are governed by linear equations for $k=0,1$, and so consist of only the seeded fundamental modes $(m, n)=(1, \pm 1)$. Their solution can be written as

$$
\left(U_{m, n}^{(k)}, V_{m, n}^{(k)}, W_{m, n}^{(k)}\right)=\left(q_{ \pm} U_{k}, q_{ \pm} V_{k}, \mp \mathrm{i} q_{ \pm} W_{k}\right)(k=0,1),
$$

where $q_{ \pm}= \pm\left(\mathrm{i} \kappa^{2} / k_{3}\right)\left(\hat{u}_{3, \pm}^{\infty} \pm \mathrm{i} \hat{u}_{2, \pm}^{\infty}\right)$, and $U_{k}, V_{k}$ and $W_{k}(k=0,1)$ are found by solving the system (B 1)-(B 8) on page 199 in LWG. Substituting the leading-order terms in the expansion (A 1) into (2.36) shows that the nonlinear terms behave as $f_{m, n}=\mathcal{O}(\bar{x}), \hat{g}_{m, n}=$ $\mathcal{O}\left(\bar{x}^{0}\right)$ and $\hat{h}_{m, n}=\mathcal{O}\left(\bar{x}^{0}\right)$ for $\bar{x} \ll 1$. Use of these estimates in (2.31)-(2.34) indicates that nonlinear effects influence $\left(U_{m, n}^{(k)}, V_{m, n}^{(k)}, W_{m, n}^{(k)}\right)$ for $k \geq 2$ and the pressure $P_{m, n}^{(1)}$. It suffices to note that $\partial P_{m, n}^{(1)} / \partial \eta=0$ for the nonlinearly generated pressure $(m, n) \neq(1, \pm 1)$.

In the region $y=\mathcal{O}(1)$, i.e. $\eta=\mathcal{O}\left((2 \bar{x})^{-1 / 2}\right)$, the governing equations follows from replacing $F$ and $F^{\prime}$ in $(2.31)-(2.34)$ by $(\eta-\beta)$ and 1 respectively, and rewriting the equations in terms of the variable $y^{(0)}$. The streamwise velocity $\hat{u}_{m, n}=0$, and the solution for $\left(\hat{v}_{m, n}, \hat{w}_{m, n}, \hat{p}_{m, n}^{(1)}\right)$ expands as power series

$$
\left(\hat{v}_{m, n}, \hat{w}_{m, n}, \hat{p}_{m, n}\right)=\sum_{k=0}^{\infty}(2 \bar{x})^{k / 2}\left(\hat{v}_{m, n}^{(k)} /(2 \bar{x})^{1 / 2}, \hat{w}_{m, n}^{(k)}, \hat{p}_{m, n}^{(k)} /(2 \bar{x})^{1 / 2}\right) .
$$

The first two terms for the velocity expansion are governed by linear equations so that the only non-zero components are seeded modes corresponding to $m=1$ and $n= \pm 1$. The solution that matches with the solutions in the outer region and the boundary layer is given by the small- $\bar{x}$ limit of (5.21)-(5.23) of LWG, namely

$$
\begin{aligned}
& \hat{v}_{1, \pm 1}^{(0)}=\frac{\mathrm{i} q_{ \pm}}{\left(\kappa_{2}-\mathrm{i}|\kappa|\right)}\left(\mathrm{e}^{\mathrm{i} \kappa_{2} y^{(0)}}-\mathrm{e}^{-|\kappa| y^{(0)}}\right), \quad \hat{w}_{1, \pm 1}^{(0)}=\frac{\mp \mathrm{i} q_{ \pm}}{\kappa_{2}-\mathrm{i}|\kappa|}\left(\kappa_{2} \mathrm{e}^{\mathrm{i} \kappa_{2} y^{(0)}}-\mathrm{i}|\kappa| \mathrm{e}^{-|\kappa| y^{(0)}}\right) \\
& \hat{p}_{1, \pm 1}^{(0)}=q_{ \pm} g_{0} \mathrm{e}^{-|\kappa| y^{(0)}}, \quad \hat{v}_{1, \pm 1}^{(1)}=q_{ \pm}|\kappa| g_{0} \mathrm{e}^{-|\kappa| y^{(0)}}, \quad \hat{w}_{1, \pm 1}^{(1)}=\mp \mathrm{i} q_{ \pm}|\kappa|^{2} g_{0} \mathrm{e}^{-|\kappa| y^{(0)}}
\end{aligned}
$$

with $g_{0}=-3 \beta /(4|\kappa|)$. Nonlinear (and viscous) effects influence the third terms in the 
velocity expansion (A 2). Therefore, the initial condition of LWG, constructed by forming the composite approximation using the first two terms in (A 1) and (A 2), remains valid to the $\mathcal{O}(\sqrt{\bar{x}})$ accuracy.

Elimination of the velocity components $\hat{v}_{m, n}^{(2)}$ and $\hat{w}_{m, n}^{(2)}$ from their governing equations in favour of the pressure shows that $\hat{p}_{m, n}^{(1)}$ satisfies the equation

$$
\left[\frac{\partial^{2}}{\partial y^{(0) 2}}-n^{2} \kappa^{2}\right] \hat{p}_{m, n}^{(1)}=\left(\epsilon / k_{1}\right) S_{m, n}
$$

after making use of (A 3), the forcing due to nonlinear interactions, i.e. $S_{m, n}$ with $(m, n) \neq$ $(1, \pm 1)$, are found as

$$
\begin{aligned}
S_{2,0} & =4\left(\kappa^{2} / \kappa_{2}^{2}\right)\left[2 \kappa_{2}^{2} \mathrm{e}^{2 \mathrm{i} \kappa_{2} y^{(0)}}+\left(\mathrm{i} \kappa_{2}-|\kappa|\right)^{2} \mathrm{e}^{\left(\mathrm{i} \kappa_{2}-|\kappa|\right) y^{(0)}}-2 \kappa^{2} \mathrm{e}^{-2|\kappa| y^{(0)}}\right], \\
S_{2, \pm 2} & =2\left(\kappa^{2} / \kappa_{2}^{2}\right)\left(\mathrm{i} \kappa_{2}+|\kappa|\right)^{2} \mathrm{e}^{\left(\mathrm{i} \kappa_{2}-|\kappa|\right) y^{(0)}} \\
S_{0,0} & =-4\left(\kappa^{2} / \kappa_{2}^{2}\right)\left[4 \kappa^{2} \mathrm{e}^{-2|\kappa| y^{(0)}}-\left(\mathrm{i} \kappa_{2}-|\kappa|\right)^{2} \mathrm{e}^{\left(\mathrm{i} \kappa_{2}-|\kappa|\right) y^{(0)}}\right]+\text { c.c. } \\
S_{0, \pm 2} & =-2\left(\kappa^{2} / \kappa_{2}^{2}\right)\left[4 \kappa^{2}+\left(\mathrm{i} \kappa_{2}+|\kappa|\right)^{2} \mathrm{e}^{\left(\mathrm{i} \kappa_{2}-|\kappa|\right) y^{(0)}}+\text { c.c. }\right] .
\end{aligned}
$$

Matching of the normal velocity $\hat{v}_{m, n}^{(2)}$ in this region with the boundary-layer solution (A 1) requires that $\hat{v}_{m, n}^{(2)}=0$ as $y^{(0)} \rightarrow 0$, since $V_{m, n}^{(1)}$ is unaffected by nonlinear effects. It follows from the $y$-momentum equation that the pressure must satisfy the boundary condition

$$
\partial \hat{p}_{m, n}^{(1)} / \partial y^{(0)}=0 \text { at } y^{(0)}=0 .
$$

The solution that satisfies the boundary condition and matches with (2.19) in the outer region is found as

$$
\left.\begin{array}{rl}
\hat{p}_{2,0}^{(1)} & =-2\left(\epsilon / k_{1}\right)\left(\kappa^{2} / \kappa_{2}^{2}\right)\left[\mathrm{e}^{2 \mathrm{i} \kappa_{2} y^{(0)}}-\mathrm{e}^{\left(\mathrm{i} \kappa_{2}-|\kappa|\right) y^{(0)}}+\mathrm{e}^{-2|\kappa| y^{(0)}}\right], \\
\hat{p}_{2, \pm 2}^{(1)} & =\left(\epsilon / k_{1}\right)\left(\kappa^{2} / \kappa_{2}^{2}\right)\left[\frac{2\left(\mathrm{i} \kappa_{2}+\kappa\right)}{\mathrm{i} \kappa_{2}-3|\kappa|} \mathrm{e}^{\left(\mathrm{i} \kappa_{2}-|\kappa|\right) y^{(0)}}+\mathcal{A}_{2, \pm 2} \mathrm{e}^{-2|\kappa| y^{(0)}}\right], \\
\hat{p}_{0,0}^{(1)} & =-4\left(\epsilon / k_{1}\right)\left(\kappa^{2} / \kappa_{2}^{2}\right)\left[\mathrm{e}^{-2|\kappa| y^{(0)}}-\mathrm{e}^{\left(\mathrm{i} \kappa_{2}-|\kappa|\right) y^{(0)}}+c . c .\right], \\
\hat{p}_{0, \pm 2}^{(1)} & =2\left(\epsilon / k_{1}\right)\left(\kappa^{2} / \kappa_{2}^{2}\right)\left[1-\frac{2\left(\mathrm{i} \kappa_{2}+\kappa\right)}{\mathrm{i} \kappa_{2}-3|\kappa|} \mathrm{e}^{\left(\mathrm{i} \kappa_{2}-|\kappa|\right) y^{(0)}}+\text { c.c. }+\mathcal{A}_{0, \pm 2} \mathrm{e}^{-2|\kappa| y^{(0)}}\right],
\end{array}\right\}
$$

where the constants

$$
\mathcal{A}_{2, \pm 2}=-\left(\kappa_{2}^{2}+\kappa^{2}\right) /\left(\mathrm{i} \kappa_{2}-3|\kappa|\right) \kappa, \quad \mathcal{A}_{0, \pm 2}=-6\left(\kappa_{2}^{2}+\kappa^{2}\right) /\left(\kappa_{2}^{2}+9 \kappa^{2}\right) .
$$

Due to the presence of the reflected perturbation, $\hat{v}_{m, n}^{(2)}$ and $\hat{w}_{m, n}^{(2)}$ are non-zero. The solution for them can easily be found by inserting (A 3) into the momentum equations, but it suffices to mention that both vanish as $y^{(0)} \rightarrow \infty$, as expected by the requirement of matching with the velocity in the outer region.

In summary, we have shown that in the upstream region corresponding to $R_{\Lambda}^{-1} \ll \bar{x} \ll$ 1 , the perturbation is linear at leading order, and the leading-order pressure is induced by the viscous displacement. Nonlinear effects produce an $\mathcal{O}(\bar{x})$ correction to the velocity, and an $\mathcal{O}(\sqrt{\bar{x}})$ correction to the pressure. The result indicates that the nonlinear regime develops gradually from the linear stage. Note that in the upstream region, the pressure plays a rather passive role within the boundary layer, with the leading-order and the nonlinearly generated pressure gradients affecting only the velocity field of $\mathcal{O}(\sqrt{\bar{x}})$ and $\mathcal{O}(\bar{x})$, respectively. 


\section{REFERENCES}

Andersson, P., Berggren, M. \& Henningson, D. S. 1999 Optimal disturbances and bypass transition in boundary layers. Phys. Fluids 11 (1), 134-150.

Andersson, P., Brandt, L., Bottaro, A. \& Henningson, D. S. 2001 On the breakdown of boundary layer streaks. J. Fluid Mech. 428, 29-60.

Arnal, D. \& Juillen, J. C. 1978 Contribution expérimental a l'etude de la receptivite d'une couche limite laminaire, a la turbulence de l'ecoulement general. CERT RT 1/5018 AYD ONERA .

Boiko, A. V., Westin, K. J. A., Klingmann, K. G. B., Kozlov, V. V. \& Alfredsson, P. H. 1994 Experiments in a boundary layer subjected to free stream turbulence. Part 2. The role of TS-waves in the transition process. J. Fluid Mech. 281, 219-245.

Bradshaw, P. 1965 The effect of wind-tunnel screens on nominally two-dimensional boundary layers. J. Fluid Mech. 22, 679-687.

Brandt, L., Cossu, C., Chomaz, J.-M., Huerre, P. \& Henningson, D. 2003 On the convectively unstable nature of optimal streaks in boundary layers. J. Fluid Mech. 485, 221-242.

Brandt, L. \& Henningson, D. S. 2002 Transition of streamwise streaks in zero-pressuregradient boundary layers. J. Fluid Mech. 472, 229-261.

Brandt, L., Schlatter, P. \& Henningson, D. S. 2004 Transition in boundary layers subject to free-stream turbulence. J. Fluid Mech. 517, 167-198.

Butler, K. \& FARrell, B. 1992 Three-dimensional optimal perturbations in viscous shear flow. Phys. Fluids 4 (8), 1637-1650.

Cossu, C. \& Brandt, L. 2002 Stabilization of Tollmien-Schlichting waves by finite amplitude optimal streaks in the Blasius boundary layer. Phys. Fluids 14 (8), L57-L60.

Cossu, C. \& Brandt, L. 2004 On Tollmien-Schlichting-like waves in streaky boundary layers. Eur. J. Mech. - B Fluids 23 (6), 815-833.

Crow, S. C. 1966 The spanwise perturbation of two-dimensional boundary-layers. J. Fluid Mech. 24, 153-164.

Dietz, A. J. 1999 Local boundary-layer receptivity to a convected free-stream disturbance. $J$. Fluid Mech. 378, 291-317.

Drazin, P. G. \& ReID, W. H. 2004 Hydrodynamic stability. Cambridge Mathematical Library.

Dryden, H. L. 1936 Air flow in the boundary layer near a plate. NACA Rep. $\mathbf{5 6 2}$.

Dryden, H. L. 1958 Combined effect of turbulence and roughness on transition. ZAMP 9, 249-258.

Duck, P. W., Ruban, A. I. \& Zhikharev, C. N. 1996 The generation of Tollmien-Schlichting waves by free-stream turbulence. J. Fluid Mech. 312, 341-371.

Ellingsen, T. \& Palm, E. 1975 Stability of linear flow. Phys. Fluids 18 (4), 487-488.

Fasel, H. \& Konzelman, U. 1990 Non-parallel stability of a flat-plate boundary layer using the complete navier-stokes equations. J. Fluid Mech. 221, 311-347.

Fransson, J., Matsubara, M. \& Alfredsson, P. H. 2005 Transition induced by free-stream turbulence. J. Fluid Mech. 527, 1-25.

Fransson, J. H. M., Brandt, L., Talamelli, A. \& Cossu, C. 2006 Delaying transition to turbulence by a passive mechanism. Phys. Rev. Lett. 96 (064501).

Gaster, M. 1962 A note on the relation between temporally-increasing and spatially-increasing disturbances in hydrodynamic stability. J. Fluid Mech. 14 (2), 222-224.

Goldstein, M. \& CHOI, S.-W. 1989 Nonlinear evolution of interacting oblique waves on twodimensional shear layers. J. Fluid Mech. 207, 97-120.

Goldstein, M. E. 1983 The evolution of Tollmein-Schlichting waves near a leading edge. J. Fluid Mech. 127, 59-81.

Goldstein, M. E. 1985 Scattering of acoustic waves into Tollmien-Schlichting waves by small streamwise variations in surface geometry. J. Fluid Mech. 154, 509-529.

Goldstein, M. E. 1997 Response of the pre-transitional laminar boundary layer to free-stream turbulence - Otto Laporte Lecture. Bull. Am. Phys. Soc. 42, 2150.

Goldstein, M. E. \& Hultgren, L. S. 1989 Boundary layer receptivity to long-wave freestream disturbances. Ann. Rev. Fluid Mech. 21, 137-166.

Goldstein, M. E. \& Leib, S. J. 1993 Three-dimensional boundary layer instability and sep- 
aration induced by small-amplitude streamwise vorticity in the upstream flow. J. Fluid Mech. 246, 21-41.

Goldstein, M. E., Leib, S. J. \& Cowley, S. J. 1992 Distortion of a flat plate boundary layer by free stream vorticity normal to the plate. J. Fluid Mech. 237, 231-260.

Goldstein, M. E. \& Sescu, A. 2008 Boundary-layer transition at high free-stream disturbance levels - beyond Klebanoff modes. J. Fluid Mech. 613, 95 - 124.

Goldstein, M. E. \& Wundrow, D. W. 1998 On the environmental realizability of algebraically growing disturbances and their relation to Klebanoff modes. Theor. Comput. Fluid Dyn. 10, $171-186$.

Hall, P. \& Horseman, N. J. 1991 The linear inviscid secondary instability of longitudinal vortex structures in boundary layers. J. Fluid Mech. 232, 357-375.

Hall, P. \& Smith, F. T. 1991 On strongly nonlinear vortex/wave interactions in boundarylayer transition. J. Fluid Mech. 227, 641-666.

Hultgren, L. \& Gustavson, L. 1981 Algebraic growth of disturbances in a laminar boundary layer. Phys. Fluids 24 (6), 1000-1004.

Jacobs, R. G. \& Durbin, P. A. 2001 Simulation of bypass transition. J. Fluid Mech. 428, $185-212$.

Jordinson, R. 1970 The flat plate boundary layer. Part 1. Numerical integration of the OrrSommerfeld equation. J. Fluid Mech. 43, 801-811.

Kachanov, Y. S. 1994 Physical mechanisms of the laminar-boundary-layer transition. Ann. Rev. Fluid Mech. 26, 411-482.

Kendall, J. M. 1991 Studies on laminar boundary layer receptivity to free-stream turbulence near a leading edge. In Boundary Layer Stability and Transition to Turbulence (ed. D.C. Reda, H. L. Reed \& R. Kobayashi). ASME FED 114, 23-30.

Kendall, J. M. 1998 Experiments on boundary-layer receptivity to free-stream turbulence. AIAA Paper 98-0530.

Kim, J., Moin, P. \& Moser, R. 1987 Turbulence statistics in fully developed channel flow at low Reynolds number. J. Fluid Mech. 177, 133-166.

Klebanoff, P. S. 1971 Effect of free-stream turbulence on a laminar boundary layer. Bull. Am. Phys. Soc. 16.

Klebanoff, P. S. \& Tidstrom, K. D. 1959 Evolution of amplified waves leading to transition in a boundary layer with zero pressure gradient. NASA TN D-195 .

LANDAhL, M. 1980 A note on an algebraic instability of inviscid parallel shear flows. J. Fluid Mech. 98, 243-251.

Leib, S. J., Wundrow, D. W. \& Goldstein, M. E. 1999 a Effect of free-stream turbulence and other vortical disturbances on a laminar boundary layer. J. Fluid Mech. 380, 169-203.

Leib, S. J., Wundrow, D. W. \& Goldstein, M. E. $1999 b$ Generation and growth of boundarylayer disturbances due to free-stream turbulence. AIAA Paper 99-0498.

LuChini, P. 1996 Reynolds-number-independent instability of the boundary layer over a flat surface. J. Fluid Mech. 327, 101-115.

LuChini, P. 2000 Reynolds-number-independent instability of the boundary layer over a flat surface: optimal perturbations. J. Fluid Mech. 404, 289-309.

Malik, M. R., Li, F., Choudhari, M. M. \& Chang, C. 1999 Secondary instability of crossflow vortices and swept-wing boundary layer transition. J. Fluid Mech. 399, 85-115.

Mans, J., De Lange, H. C. \& van Steenhoven, A. A. 2007 Sinuous breakdown in a flat plate boundary layer exposed to free-stream turbulence. Phys. Fluids 19 (088101).

Matsubara, M. \& Alfredsson, P. H. 2001 Disturbance growth in boundary layers subjected to free-stream turbulence. J. Fluid Mech. 430, 149-168.

Nagarajan, S., Lele, S. K. \& Ferziger, J. H. 2007 Leading-edge effects in bypass transition. J. Fluid Mech. 572, 471-504.

Ovchinnikov, V., Choudhari, M. M. \& Piomelli, U. 2008 Numerical simulations of boundary-layer bypass transition due to high-amplitude free-stream turbulence. J. Fluid Mech. 613, 135-169.

Pope, S. B. 2000 Turbulent Flows. Cambridge University Press.

Ricco, P. 2009 The pre-transitional Klebanoff modes and other boundary layer disturbances induced by small-wavelength free-stream vorticity. J. Fluid Mech. 638, 267-303. 
Ricco, P. \& Wu, X. 2007 Response of a compressible laminar boundary layer to free-stream vortical disturbances. J. Fluid Mech. 587, 97-138.

Ruban, A. I. 1985 On the generation of Tollmien-Schlichting waves by sound. Fluid Dyn. 25(2), 213-221.

Saric, W. S., Reed, H. L. \& Kerschen, E. J. 2002 Boundary-layer receptivity to freestream disturbances. Ann. Rev. Fluid Mech. 34, 291.

Schlatter, P., Brandt, L., De Lange, H. C. \& Henningson, D. S. 2008 On streak breakdown in bypass transition. Phys. Fluids 20 (101505).

Schubauer, G. \& Skramstad, H. 1947 Laminar boundary-layer oscillations and transition on a flat plate. NACA TN 909 .

TAYLOR, G. I. 1939 Some recent developments in the study of turbulence. Fifth Intl. Congr. for Appl. Mech. (ed. J.P. Den Hartog 8 Peters) - Wiley/Chapman and Hall, New York-London pp. 294-310.

Theofilis, V. 2003 Advances in global linear instability analysis of non-parallel and threedimensional flows. Prog. Aerospace Sciences 39, 249-315.

Trefethen, L., Trafethen, A., Reddy, S. \& Driscoll, T. 1993 Hydrodynamic stability without eigenvalues. Science 261, 578-584.

Tumin, A. 2001 A model of spatial algebraic growth in a boundary layer subjected to a streamwise pressure gradient. Phys. Fluids 13 (5), 1521-1523.

Westin, K. J. A., Boiko, A. V., Klingmann, B. G. B., Kozlov, V. V. \& Alfredsson, P. H. 1994 Experiments in a boundary layer subjected to free stream turbulence. Part 1. Boundary layer structure and receptivity. J. Fluid Mech. 281, 193-218.

Wu, X. 2001a On local boundary-layer receptivity to vortical disturbances in the free-stream. J. Fluid Mech. 449, 373-393.

Wu, X. $2001 b$ Receptivity of boundary layers with distributed roughness to vortical and acoustic disturbances: a second-order asymptotic theory and comparison with experiments. J. Fluid Mech. 431, 91-133.

Wu, X. \& Choudhari, M. 2003 Linear and non-linear instabilities of a Blasius boundary layer perturbed by streamwise vortices. Part 2 . Intermittent instability induced by longwavelength Klebanoff modes. J. Fluid Mech. 483, 249-286.

Wu, X., Lee, S. S. \& Cowley, S. J. 1993 On the weakly nonlinear three-dimensional instability of shear layers to pairs of oblique waves: the Stokes layer as a paradigm. J. Fluid Mech. 253, 681-721.

Wu, X. \& Luo, J. 2003 Linear and non-linear instabilities of a Blasius boundary layer perturbed by streamwise vortices. Part 1. Steady streaks. J. Fluid Mech. 483, 225-248.

Wundrow, D. W. \& Goldstein, M. E. 2001 Effect on a laminar boundary layer of smallamplitude streamwise vorticity in the upstream flow. J. Fluid Mech. 426, 229-262.

ZAKi, T. \& Durbin, P. A. 2006 Continuous mode transition and the effects of pressure gradient. J. Fluid Mech. 563, 357-388.

Zuccher, S., Bottaro, A. \& Luchini, P. 2006 Algebraic growth in a Blasius boundary layer: Nonlinear optimal disturbances. Eur. Jour. Mech. - B/Fluids 25 (1), 1-17. 

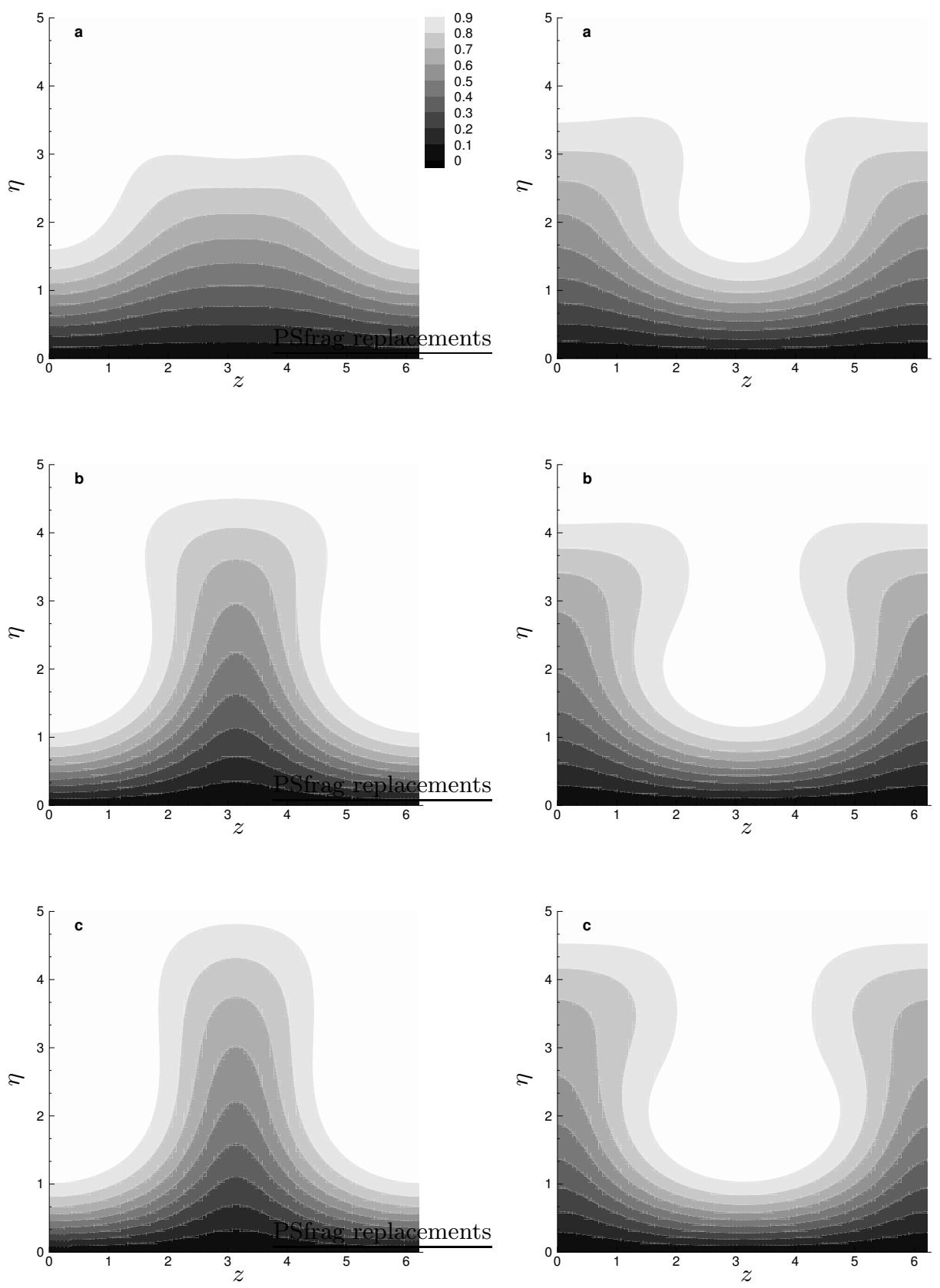

Figure 12. Contour of instantaneous streamwise velocity in $\eta-z$ plane. Left: $\bar{x}=0.7$ at phases $\phi=25 \pi / 16(\mathrm{a}), 30 \pi / 16(\mathrm{~b})$ and $31 \pi / 16(\mathrm{c})$. Right: $\bar{x}=1.2$ at phases $\phi=13 \pi / 16(\mathrm{a}), \pi(\mathrm{b})$ and $5 \pi / 4(\mathrm{c})$. 

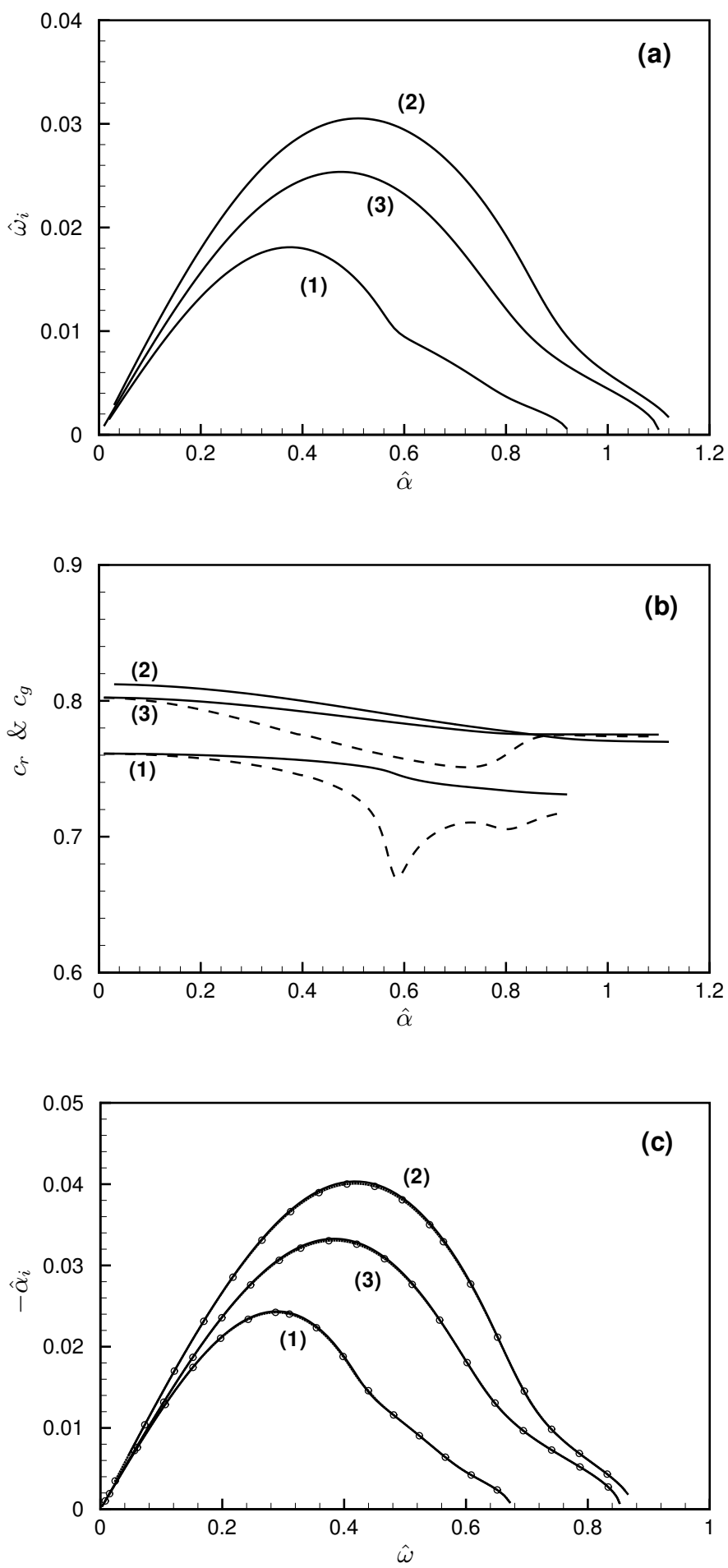

FiguRE 13. Streak instability characteristics at $\bar{x}=0.7$ : (a) Temporal growth rate $\hat{\omega}_{i}=\hat{\alpha} c_{i}$ v.s. $\hat{\alpha}$; (b) The phase speed $c_{r}$ (solid lines) and group velocity $c_{g}$ (dashed lines) v.s. $\hat{\alpha}$; (c) Spatial grow rate $\hat{\alpha}_{i}$ v.s. $\hat{\omega}$ with symbols representing the result obtained by using (5.6). The curves (1), (2) and (3) correspond to the phases $\phi=25 \pi / 16,15 \pi / 8,31 \pi / 16$, respectively. 

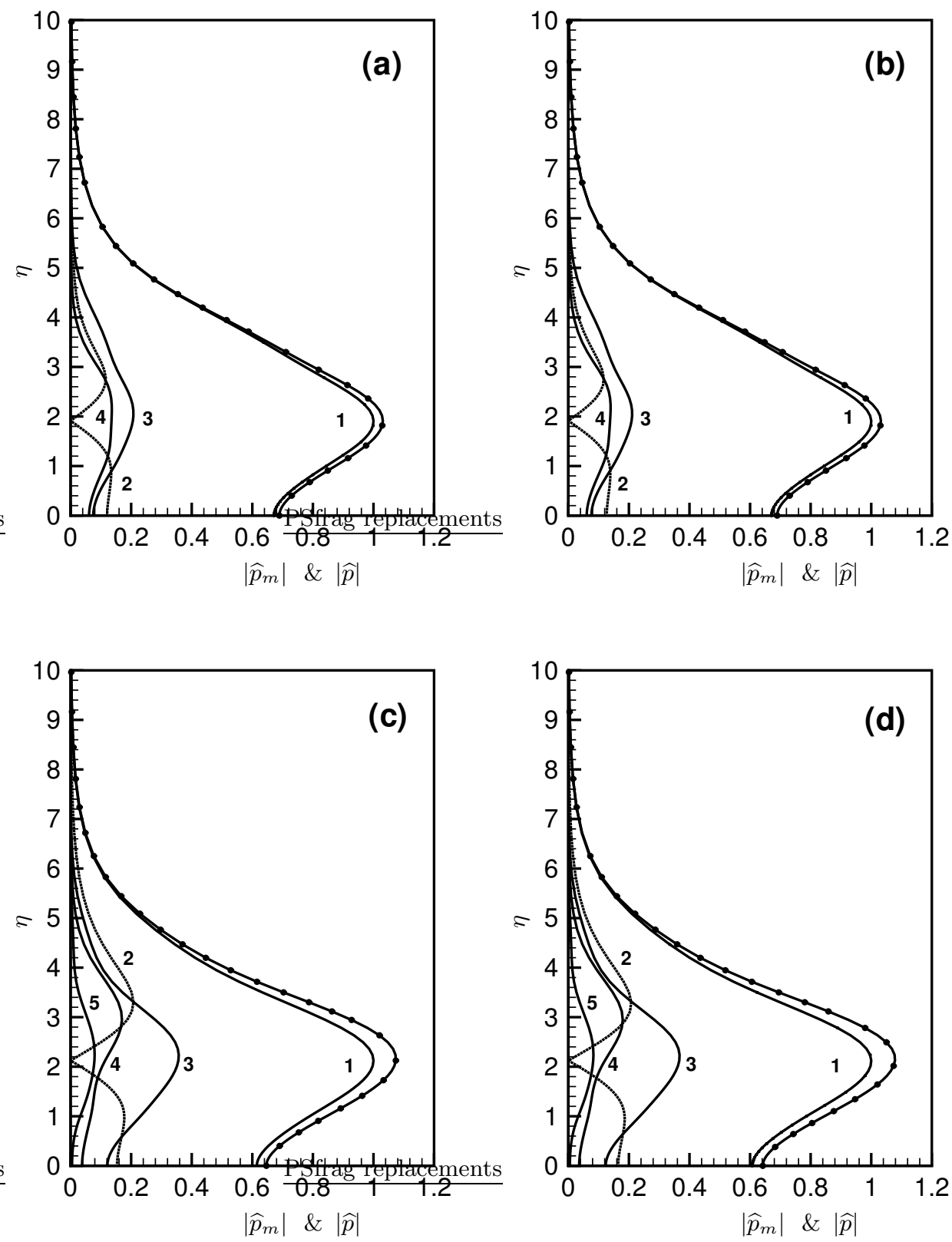

FiguRE 14. Eigenfunctions of the most unstable modes at $\bar{x}=0.7$ : (a) $\phi=25 \pi / 16$, temporal mode $\hat{\alpha}=0.375$; (b) $\phi=25 \pi / 16$, spatial mode $\hat{\omega}=0.29$; (c) $\phi=15 \pi / 8$, temporal mode $\hat{\alpha}=0.5$; (d) $\phi=15 \pi / 8$, spatial mode $\hat{\omega}=0.42$. The solid lines with symbols represent $|\widehat{p}| \equiv \sum\left|\widehat{p}_{m}\right|$. 

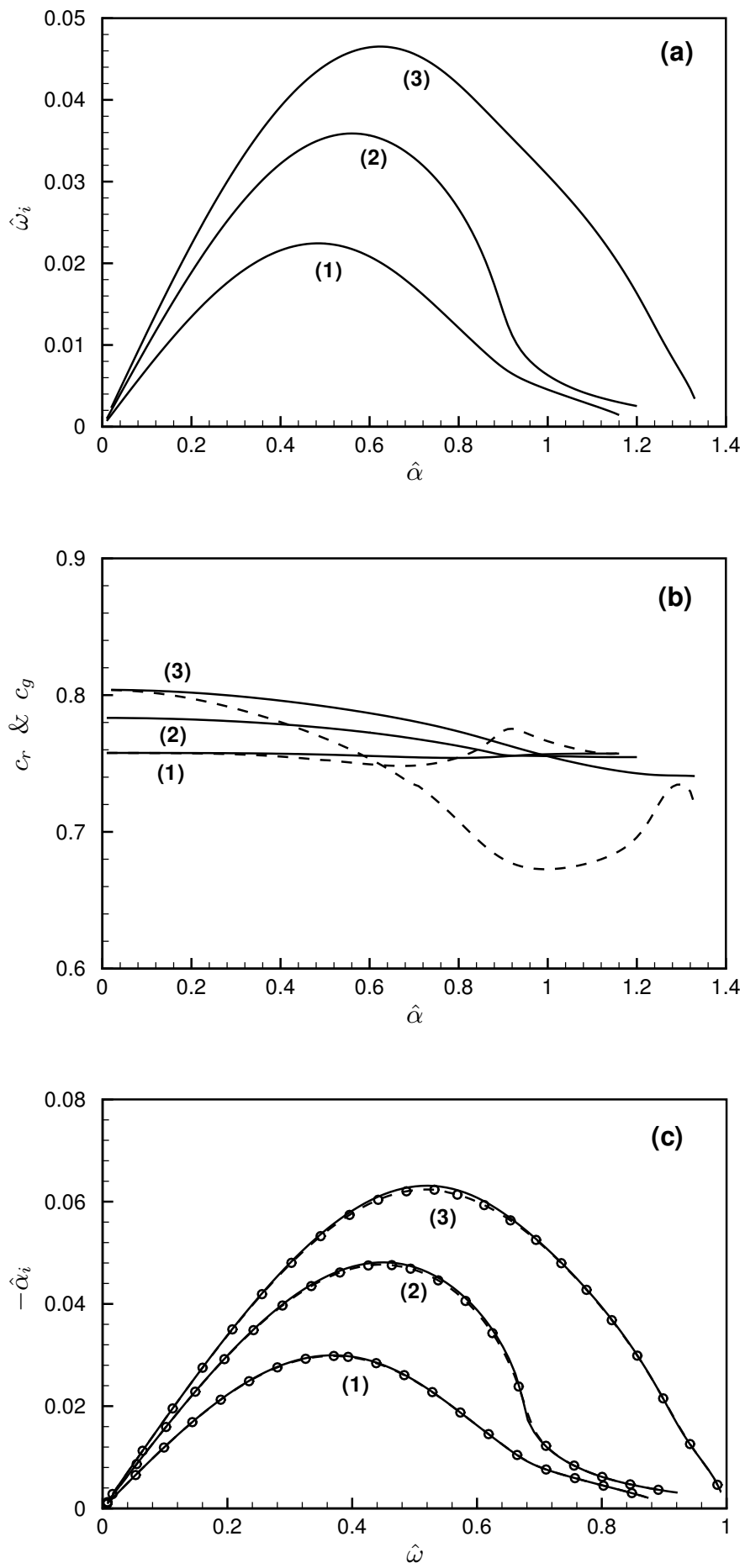

FiguRE 15. Streak instability characteristics at $\bar{x}=1.2$ : (a) Temporal growth rate $\hat{\omega}_{i}=\hat{\alpha} c_{i}$ v.s. $\alpha$; (b) The phase speed $c_{r}$ (solid lines) and group velocity $c_{g}$ (dashed lines) v.s. $\hat{\alpha}$; (c) Spatial grow rate $\hat{\alpha}_{i}$ v.s. $\hat{\omega}$ with symbols representing the result obtained by using (5.6). The curves (1), (2) and (3) correspond to the phases $\phi=13 \pi / 16, \pi, 5 \pi / 4$, respectively. 

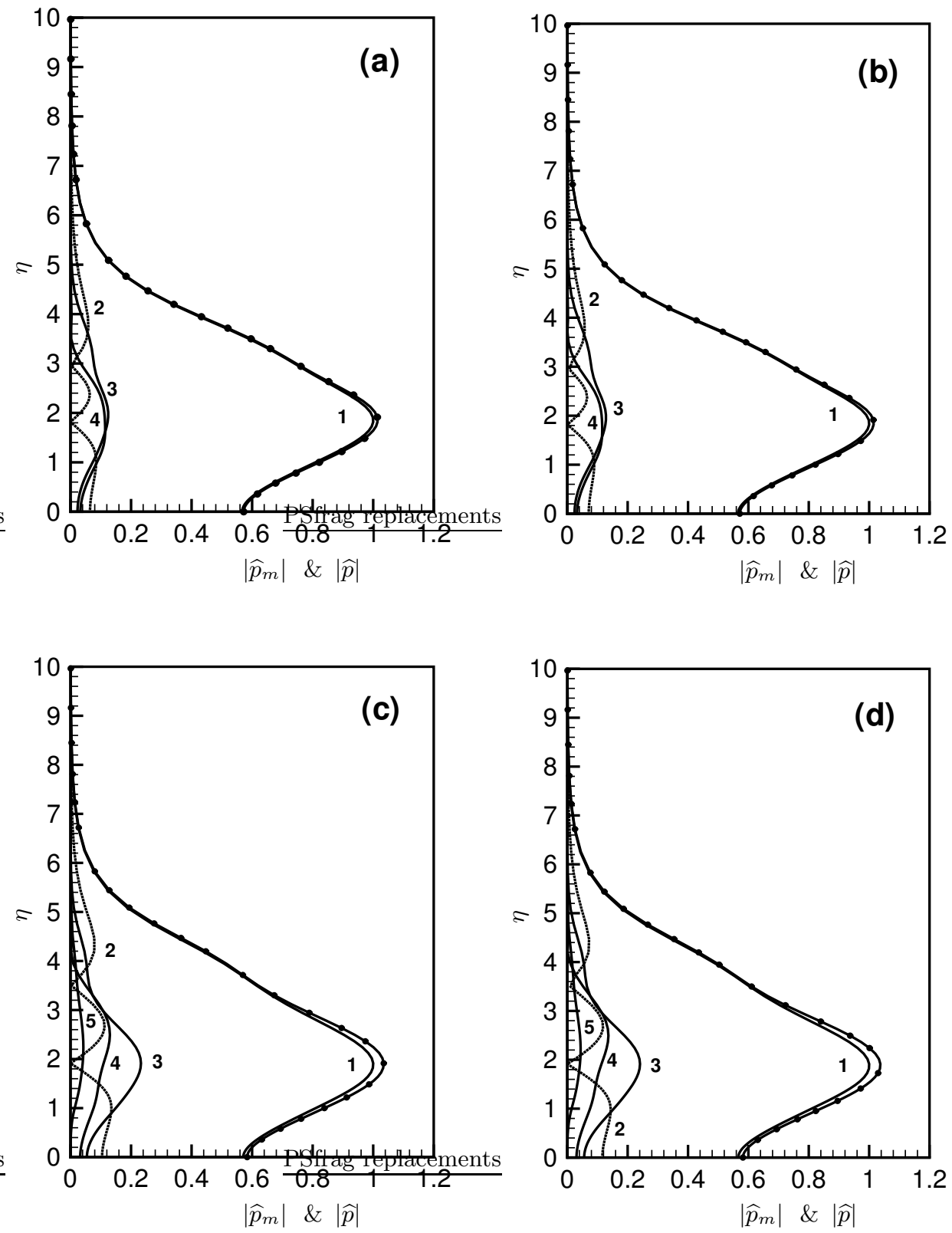

FigURE 16. Eigenfunctions of the most unstable modes at $\bar{x}=1.2$ : (a) $\phi=13 \pi / 16$, temporal mode $\hat{\alpha}=0.48$; (b) $\phi=13 \pi / 16$, spatial mode $\hat{\omega}=0.37$; (c) $\phi=5 \pi / 4$, temporal mode $\hat{\alpha}=0.56$; (d) $\phi=5 \pi / 4$, spatial mode $\hat{\omega}=0.45$. The solid lines with symbols represent $|\widehat{p}| \equiv \sum\left|\widehat{p}_{m}\right|$. 

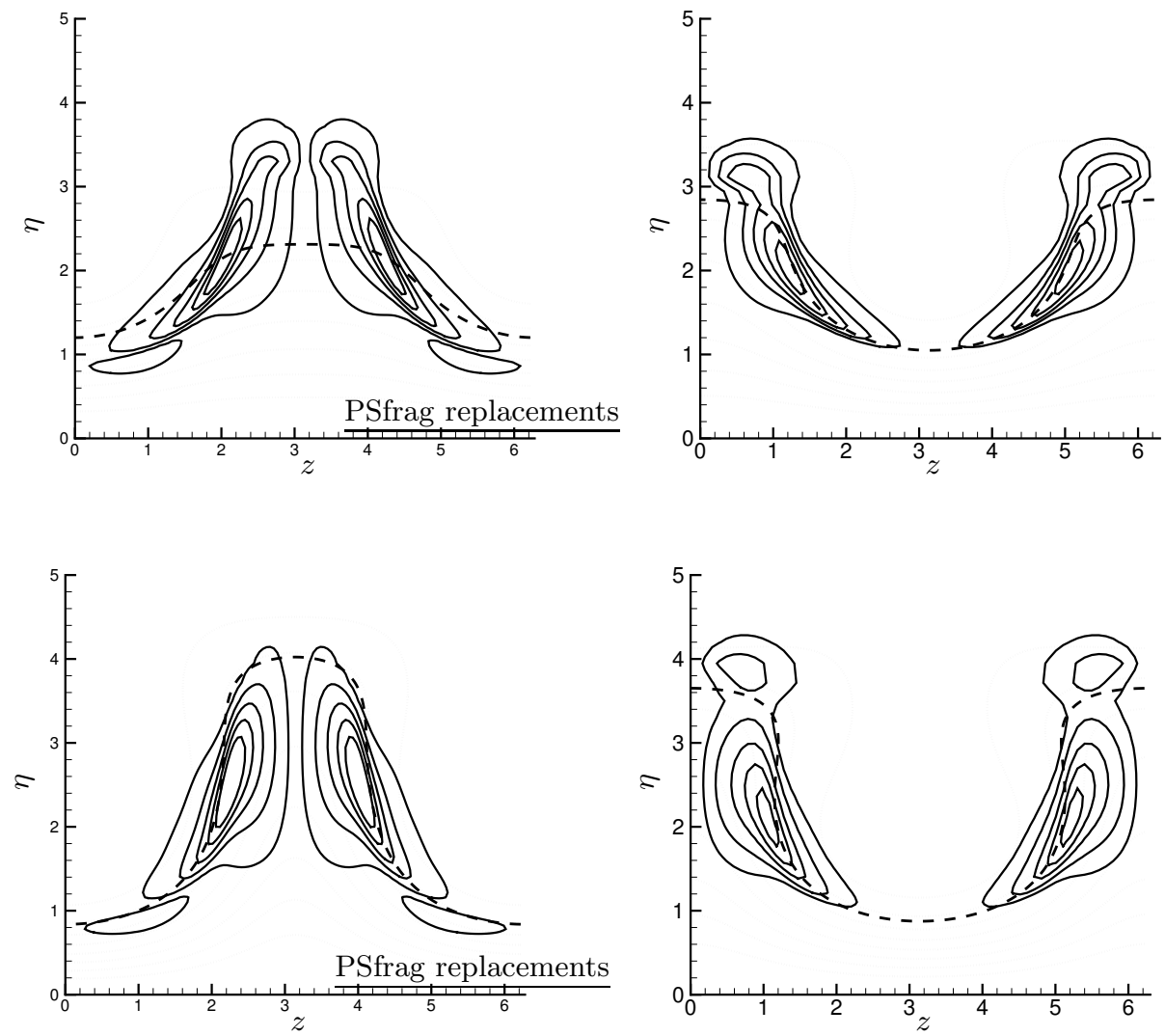

Figure 17. Contours of the eigenfunction for the streamwise velocity $\widehat{u}$. Left: $\bar{x}=0.7$ and $\phi=25 \pi / 16,15 \pi / 8$. Right: $\bar{x}=1.2$ and $\phi=13 \pi / 16, \pi$. The light dashed lines are contours of the instantaneous velocity of the base flow $U(\eta, z ; \bar{x}, t)$, and the thick dashed lines represent the critical level $\eta_{c}$, at which $U\left(\eta_{c}, z\right)=c_{r}$. 